\title{
Non-linear response of a Kondo system: Perturbation approach to the time dependent Anderson impurity model
}

\author{
Y. Goldin and Y. Avishai* \\ Physics Department, Ben Gurion University of the Negev \\ Beer Sheva, Israel
}

(June 21, 2017)

\begin{abstract}
Nonlinear tunneling current through a quantum dot (an Anderson impurity system) subject to both constant and alternating electric fields is studied in the Kondo regime. A systematic diagram technique is developed for perturbation study of the current in physical systems out of equilibrium governed by time - dependent Hamiltonians of the Anderson and the Kondo models. The ensuing calculations prove to be too complicated for the Anderson model, and hence, a mapping on an effective Kondo problem is called for. This is achieved by constructing a time - dependent version of the Schrieffer - Wolff transformation. Perturbation expansion of the current is then carried out up to third order in the Kondo coupling $J$ yielding a set of remarkably simple analytical expressions for the current. The zero - bias anomaly of the direct current $(D C)$ differential conductance is shown to be suppressed by the alternating field while side peaks develop at finite source - drain voltage. Both the direct component and the first harmonics of the time - dependent response are equally enhanced due to the Kondo effect, while amplitudes of higher harmonics are shown to be relatively small. A "zero alternating bias anomaly" is found in the alternating current $(A C)$ differential conductance, that is, it peaks around zero alternating bias. This peak is suppressed by the constant
\end{abstract}


bias. No side peaks show up in the differential alternating - conductance but their counterpart is found in the derivative of the $A C$ with respect to the direct bias. The results pertaining to nonlinear response are shown to be valid also below the Kondo temperature.

Typeset using REVTEX 


\section{INTRODUCTION}

An attractive research direction in contemporary condensed matter physics seems to be the study of non-equilibrium many - body phenomena. A promising experimental and theoretical framework for investigating this topic is provided by the physics of quantum dot systems. The reason for that is clear, namely, quantum dots are fabricated and their properties can be elucidated by present day experimental techniques. Indeed, recent experiments on electron transport in quantum dots at low temperatures reveal signatures of coherent many - body physics such as the emergence of zero bias anomaly in current - voltage characteristics 1 目 which is due to the formation of a many body resonance. At the same time, the underlying theoretical models are of sufficient simplicity, so that one encounters a rare occasion where one has an experimentally accessible non-equilibrium quantum system that is also amenable to reliable and controllable theoretical approaches.

So far, the main effort in the physics of quantum dots has been devoted to the study of different phenomena emerging from the presence of large Coulomb interaction in the dot. In this contexts, the most familiar and simplest topic is the Coulomb blockade. Its essence is simply encoded as a capacitance effect: every extra electron coming into the dot has to overcome a charging energy $e^{2} / C$, where $C$ is the capacitance of the dot. If the gate voltage is not tuned to supply this energy, the tunneling is (Coulomb) blocked. Yet, we believe that essentially genuine many - body aspects of resonant tunneling might be better revealed in the physics that goes substantially beyond the simplified Coulomb blockade picture. By this we mean the Kondo effect and other facets of strongly correlated electronic systems. They result from an intricate combination of electron - electron interaction and tunneling.

Whereas the Kondo effect in bulk materials has been a thought - inspiring subject of research for more than three decades (for review, see Ref. 4.5), its emergence in quantum dot physics appears to be relatively new. Yet, it proves to be equally thought - inspiring. In particular, it opens a road to explore the non-equilibrium Kondo physics. Its hallmark is the zero - bias anomaly, that is, an appearance of a large narrow peak in the differential 
conductance around zero bias. Pertinent experiments have been carried out on crossed wire tungsten junctions 6 , quenched lithographic point contacts break junctions 910 and, recently, quantum dotst

In parallel, progress has also been recorded in numerous theoretical works, 1124 . Among other directions of research, interest is focused on time - dependent aspects of the Kondo physics 1720 24. Although an actual experimental research has not yet been carried out, measurement of alternating tunneling current in the appropriate range of frequencies proves to be feasible 25 28,29. Moreover, application of an external alternating electric field nontrivially affects the $d c$, an observable which can actually be measured $30-34$. We hope that the pertinent experiments can be carried out in the near future.

It is useful to briefly mention the main features of the Kondo effect in bulk systems and in quantum dots. The Kondo effect was first revealed in the early 30's as an enhancement in the resistivity of certain metals with decreasing temperature. Being a puzzle for thirty years it was then attributed by Kondo 35 to interaction between conducting electrons and magnetic impurity atoms. Subsequent investigations proved that at low temperatures a hybrid state is formed consisting of the conducting electrons assembled around an impurity so that at zero temperature the magnetic moment of the impurity is completely screened.

Within the realm of quantum dot physics, the impurity spin is represented by the spin of a single electron which is virtually locked in a deep level of the quantum dot. The formation of the hybrid state (either in bulk systems or in quantum dots) is accompanied by an appearance of a narrow peak in the interacting density of states of the impurity (or dot electron) close to the chemical potential of the band (lead) electrons. This peak is termed as an "Abrikosov - Suhl resonance" or "Kondo resonance" (see Ref. 田, pp.109, $127-132,210)$. For quantum dots in a static non-equilibrium situation, when two chemical potentials are present in the system (one for every lead), the pertinent peak in the interacting density of states splits, under certain conditions, into two peaks, one at each chemical potential13, 16, 18, 19, 36 (see Fig. [1a).

The main object of our study in the present work is the response of a quantum dot to the 
application of a strong time - dependent external electric field. For simplicity it is assumed that the field is monochromatic, whose frequency $\Omega$ is in the range of tens of gigahertz. The relevant Physics implies an interesting extension of the underlying Kondo physics since, in a time - dependent field, every eigenstate of electrons in the leads is split 37,38 into a family of states whose (quasi) energies are separated by the photon energy $\hbar \Omega$. Consequently, there is a family of Fermi seas, and, correspondingly, a family of chemical potentials in each lead. Thus, the Kondo peak in the density of states might split into a whole set of peaks (see Fig. 11b). Numerical calculations of the $D d \sqrt{17}$ indicate that this is indeed the case. We think that the occurrence of many separated Abrikosov-Suhl resonances can be tested experimentally. It will show up as side peaks in the (otherwise monotonic) $I(V)$ curve displaying the zero bias anomaly of the direct current. Another interesting question is how the contributions of these peaks to the current, which are mutually coherent, interfere. This interference can show up only in the time - dependent current 29.

In this work, both direct and alternating tunneling currents are calculated within a perturbation scheme specially adapted for systems out of equilibrium. So far, calculations of the current through a Kondo system subject to a time - dependent bias were carried out using various assumptions and approximations. Here we carry out straightforward nonequilibrium perturbation expansion of the current in powers of the coupling constant between the quantum dot (or impurity atom) and the electrons in the conduction bands. Indeed, perturbation theory proved its usefulness in calculations related to the equilibrium Kondo model in bulk systems. $35,39,40$. Recently it has been used for quantum dots described by the Anderson model (in the Kondo regime) with a constant voltage bias 18 .

Let us then briefly list the main achievements of the present study. Our formulation starts from the time - dependent Anderson model which has already been shown to adequately describe the essential physics of a quantum dot (or a tunneling system based on an impurity atom) at voltages and frequencies less than the level spacing in the tunneling region. We then develop the pertinent diagram perturbation technique which is able to treat non-equilibrium tunneling problems such as the time - dependent Anderson and 
Kondo models (for the former one it employs the slave - bosons method). A key point in the derivation is provided by combination of a specific approach suggested by Coleman 11 to perform self consistent quantum and thermodynamical averaging in strongly correlated systems, with the Schwinger - Keldysh non-equilibrium Green functions formalism. As it turn out, calculations pertaining to the Anderson model appear to be rather cumbersome. At this stage we are therefore content by stating the rules for calculating diagrams and by pointing out a specific example where the Kondo behavior shows up. At the same time, we find it more practical to map the original time - dependent Anderson Hamiltonian on an effective time - dependent Kondo Hamiltonian. This is achieved by introducing a time dependent Schrieffer - Wolff transformation. Perturbation expansion of the current is then carried out within the Kondo model up to third order in the Kondo coupling $J$ (sixth order in the tunneling coupling between conducting electrons and the dot). Remarkably simple analytical expressions are obtained for the whole spectrum of the tunneling current. It is shown that the zero - bias anomaly of the $D C$ differential conductance is, in general, suppressed by an external alternating field, while side peaks develop at higher source drain bias. The nonlinear time - dependent current is found to be an interference sum of "direct like" contributions, each one with an effective bias determined by the number of absorbed or emitted photons. The interference is shown to be rather destructive for all higher harmonics except the direct and the first ones. These two harmonics (and only them) are enhanced as a result of the Kondo effect while the other harmonics remain relatively small. In this sense the Kondo system behaves like a usual resistor (although the current is Kondo enhanced). Namely, direct and first harmonic input voltage lead to a direct and first harmonic current response. This result is demonstrated to be remarkably different from that for a non - interacting one - level system where all the harmonics emerge together. Moreover, it is shown to be valid also below the Kondo temperature. Finally, we also calculate the differential alternating conductance and find that like its direct analog it displays a zero alternating bias anomaly. Inspecting the dependence of the $A C$ on the direct bias reveals a non - trivial structure marked by side peaks. 
The rest of the paper is organized as follows. In the next section, diagrammatic rules for the non-equilibrium perturbation expansion of the time - dependent Anderson model in the slave - boson representation are formulated. The diagrams are drawn up to sixth order in the tunneling coupling between the leads and the dot $\left(\tilde{V}_{k d}\right)$ and some formal analytical expressions for the current are obtained. Further elaboration of the perturbation expansion in this model meets computational problems which today look severe. In section 【II a time dependent version of the Schrieffer - Wolff transformation is developed in order to transform the time dependent Anderson model onto a Kondo - type model. In section IV perturbation expansion of the current (in the time - dependent Kondo model) is carried out in powers

of the coupling strength $\left(\tilde{J}_{k^{\prime} k}\right)$. An analytical expression for the current is obtained and the novel results arising from that expression are discussed. The paper is concluded with a summary which, in particular, includes some prospects for further research directions. In view of the inherent complexity of the pertinent formulation, we try to present it in a pedagogical style.

\section{PERTURBATION EXPANSION OF THE CURRENT IN THE TIME-DEPENDENT ANDERSON MODEL}

\section{A. Bare Hamiltonian and parameter specification}

In this section we first introduce the time - dependent Anderson Hamiltonian and specify the range of parameters appropriate for the pertinent physical problem. Then we recall the slave - boson approach to the Anderson model and combine it with the Schwinger - Keldysh non-equilibrium Green function formalism in order to get an equation for the tunneling current. Then we develop a perturbation expansion of the current in powers of the tunneling strength $\left(\tilde{V}_{k d}\right)$ up to sixth order in which the Kondo physics above the Kondo temperature (or in a strong enough external field) is unraveled.

The time - dependent Anderson Hamiltonian takes the form, 
$\tilde{H}_{A}=\sum_{k \in L, R ; \sigma}\left(\epsilon_{k}+\Delta_{L(R)}(t)\right) a_{k, \sigma}^{\dagger} a_{k, \sigma}+\sum_{\sigma} \epsilon_{d, \sigma} c_{d, \sigma}^{\dagger} c_{d, \sigma}+\frac{1}{2} U \sum_{\sigma, \sigma^{\prime} \neq \sigma} n_{\sigma} n_{\sigma^{\prime}}+\sum_{k \in L, R ; \sigma}\left(\tilde{V}_{k d} a_{k, \sigma}^{\dagger} c_{d, \sigma}+\right.$ h.c. $)$.

Here $a_{k, \sigma}^{\dagger}\left(a_{k, \sigma}\right)$ creates (annihilates) an electron with momentum $k$ and spin $\sigma$ in the left $(k \in L)$ or the right $(k \in R)$ lead, $c_{d, \sigma}^{\dagger}\left(c_{d, \sigma}\right)$ creates (annihilates) an electron with spin $\sigma$ in the dot, $\epsilon_{k}$ and $\epsilon_{d, \sigma}$ are single - particle energies in the leads and the dot respectively, $U$ is the Coulomb interaction energy in the dot and $n_{\sigma} \equiv c_{d, \sigma}^{\dagger} c_{d, \sigma}$. The transfer matrix elements $\tilde{V}_{k d}$ between the leads and the dot are assumed to be small compared with $\epsilon_{d, \sigma}$ and $U$. The external fields are included through potential shifts of the leads $\Delta_{L(R)}(t)$, defined as,

$$
\Delta_{L(R)}(t) \equiv \phi_{L(R)}+W_{L(R)} \cos \left(\Omega t+\alpha_{L(R)}\right)
$$

The first term above describes a constant potential bias, while the second one is due to an alternating field, which, for simplicity, is assumed to be monochromatic. We note that the chemical potentials in the leads are shifted by the same amount $\Delta_{L(R)}(t)$ as the single-particle energies, hence the population of energy levels in the leads remains intact.

As far as the value of the parameters is concerned, our attention here is focused on the Kondo regime which is determined by the conditions,

$$
\epsilon_{d, \sigma}<0, \quad \epsilon_{d, \sigma}+U>0, \quad\left|\epsilon_{d, \sigma}\right|, \epsilon_{d, \sigma}+U>\Gamma_{\sigma}
$$

where $\Gamma_{\sigma}=2 \pi \sum_{k \in L, R}\left|\tilde{V}_{k d}\right|^{2} \delta\left(\epsilon_{d, \sigma}-\epsilon_{k}\right)$ are the widths of the energy levels in the dot. Furthermore, it is assumed that the external fields are not strong enough to draw the system out of this regime, so that:

$$
\begin{gathered}
\left|\phi_{L(R)}\right|<\left|\epsilon_{d, \sigma}\right|, \epsilon_{d, \sigma}+U \text { and } \\
\Omega, W_{L}, W_{R}<\left|\epsilon_{d, \sigma}\right|, \epsilon_{d, \sigma}+U .
\end{gathered}
$$

It should be stressed however that these conditions do not imply a linear response regime. The latter is defined by the conditions $\left|\phi_{L(R)}\right|, W_{L(R)} \ll T$ while $T \ll\left|\epsilon_{d, \sigma}\right|, \epsilon_{d, \sigma}+U$. 
At this point it is convenient to apply a canonical transformation 45.46 on the Anderson model (11) whose purpose is to transfer the dependence on time into that part which contains a small parameter. The transformation is defined as follows:

$$
\begin{aligned}
H_{A} & =\mathcal{U}^{-1} \tilde{H}_{A} \mathcal{U}-\mathcal{U}^{-1} \frac{\partial}{\partial t} \mathcal{U} \\
\mathcal{U}(t) & =\exp \left\{\frac{i}{\hbar} \int_{-\infty}^{t} d t_{1}\left[\Delta_{L}\left(t_{1}\right) N_{L}+\Delta_{R}\left(t_{1}\right) N_{R}\right]\right\} .
\end{aligned}
$$

where $\hat{N}_{L(R)} \equiv \sum_{\sigma, k \in L(R)} a_{k, \sigma}^{\dagger}(t) a_{k, \sigma}(t)$. The Hamiltonian $H_{A}$ resulting from this transformation reads,

$$
\begin{aligned}
H_{A}= & \sum_{k \in L, R ; \sigma} \epsilon_{k} a_{k, \sigma}^{\dagger} a_{k, \sigma}+\sum_{\sigma} \epsilon_{d, \sigma} c_{d, \sigma}^{\dagger} c_{d, \sigma}+\frac{1}{2} U \sum_{\sigma, \sigma^{\prime} \neq \sigma} n_{d, \sigma} n_{d, \sigma^{\prime}}+ \\
& +\sum_{k \in L, R ; \sigma}\left(V_{k d}(t) a_{k, \sigma}^{\dagger} c_{d, \sigma}+\text { h.c. }\right) .
\end{aligned}
$$

where

$$
V_{k d}(t)=\tilde{V}_{k d} \exp \left\{\frac{i}{\hbar} \int_{-\infty}^{t} d t_{1} \Delta_{L(R)}\left(t_{1}\right)\right\}
$$

Usually, in tunneling systems the barriers have low transparency. Therefore it is convenient to consider the tunneling part of the Hamiltonian, i.e. $\sum_{k \in L, R ; \sigma}\left(V_{k d}(t) a_{k, \sigma}^{\dagger} c_{d, \sigma}+\right.$ h.c. $)$, as a perturbation 47 . It is well known, however, that if the interaction $\frac{1}{2} U \sum_{\sigma, \sigma^{\prime} \neq \sigma} n_{d, \sigma} n_{d, \sigma^{\prime}}$ is kept in the "unperturbed" Hamiltonian the Wick's theorem can not be applied. In order to circumvent this problem we assume that the energies associated with the direct and alternating voltages as well as the pertinent frequencies are smaller than the Coulomb interaction energy $U$ in the dot. In other words, the assumption $U \rightarrow \infty$ should be an excellent approximation. We then apply the method of slave (auxiliary) bosons 41,42 using a certain version of it which is due to Colemant 11 . Accordingly, the ordinary electron operators in the $\operatorname{dot} c_{d, \sigma}, c_{d, \sigma}^{\dagger}$, which transform a singly occupied state into an empty one and vise versa, are factored into a boson operator and a fermion operator 16 :

$$
\begin{aligned}
& c_{d, \sigma}=b^{\dagger} f_{\sigma} \\
& c_{d, \sigma}^{\dagger}=b f_{\sigma}^{\dagger} .
\end{aligned}
$$


The slave boson operator $b^{\dagger}(b)$ creates (annihilates) an empty state, while the slave fermion operator $f_{\sigma}\left(f_{\sigma}^{\dagger}\right)$ annihilates (creates) a singly occupied state. In this representation the Hamiltonian (6) becomes,

$$
H_{S B}=\sum_{k \in L, R ; \sigma} \epsilon_{k} a_{k, \sigma}^{\dagger} a_{k, \sigma}+\sum_{\sigma} \epsilon_{d, \sigma} f_{\sigma}^{\dagger} f_{\sigma}+\sum_{\sigma, k \in L, R}\left(V_{k d}(t) a_{k, \sigma}^{\dagger} b^{\dagger} f_{\sigma}+\text { h.c. }\right) \text {. }
$$

The Coulomb interaction does not appear in the Hamiltonian any more. Indeed, when $U$ is infinite it completely eliminates the possibility of double occupancy of the dot. This projection is accomplished by including the annihilation operator $b$ in equations (8), which prevents creation of the doubly occupied state, and the constraint that the total number of slave bosons and slave fermions,

$$
Q_{A} \equiv b^{\dagger} b+\sum_{\sigma} f_{\sigma}^{\dagger} f_{\sigma}
$$

must be equal to unity. The operator $Q_{A}$ commutes with the Hamiltonian (9) so that the "charge" $Q_{A}$ is not changed during the evolution of the system. Therefore the requirement

$Q_{A}=1$ does not really constrain the dynamics of the system. Rather, it assures that the initial state does not contain a doubly occupied state as a component. In order to enforce the condition $Q_{A}=1$ Coleman 41 introduced a Lagrange multiplier (chemical potential) $-\lambda$ and considered the Hamiltonian $H_{S B}+\lambda Q_{A}$. The calculations are to be done with finite $\lambda$ and then, at the end, $\lambda \rightarrow \infty$. If the tunneling part of $H_{S B}+\lambda Q_{A}$ is chosen as perturbation the remaining "unperturbed" Hamiltonian is now quadratic in the creation and annihilation operators. The Wick's theorem can then be applied and diagrammatic expansion is feasible. We notice that the unperturbed part has become a simple time - independent free - particle Hamiltonian while the perturbation (tunneling part) contains both interaction and time dependence.

\section{B. The tunneling current}

The tunneling current from the left (right) lead into the central region is defined as the product of electron charge $(-e)$ and the rate of change in the number of electrons in 
that lead. The latter is obtained by commutating the number - of - electrons (Heisenberg) operator $\hat{N}_{L(R)}$ with the Hamiltonian (9). This yields

$$
I_{L(R)}^{T}(t)=-e\left\langle\frac{d \hat{N}_{L(R)}(t)}{d t}\right\rangle=-\frac{i e}{\hbar} \sum_{\sigma, k \in L(R)} V_{k d}(t) \operatorname{Tr}_{F_{1}^{A}}\left\{\rho_{F_{1}^{A}}(0) a_{k, \sigma}^{\dagger}(t) b^{\dagger}(t) f_{\sigma}(t)\right\}+c . c .
$$

where $\rho_{F_{1}^{A}}(0)$ is the density matrix of the system at a certain fixed time which is taken here at $t=0$. Generally, it should include all the changes that the system has undergone since the tunneling was switched on 50 . We recall that the above equation is written in the Heisenberg representation. The angle brackets on the left hand side mean, of course, quantum average over the physical Hilbert space. At infinite $U$ it is only a subspace $F_{1}^{A}$ of the full Hilbert space for the slave - boson Hamiltonian (9) which is determined by the condition $Q_{A}=1$. It is explicitly manifested on the right - hand side of the above expression by the subscript $F_{1}^{A}$ which restricts the trace to the physical subspace $F_{1}^{A}$. In the following we calculate directly the current (11) without prior elaboration on the dot Green functions as it was done in Refs. 11, 13, 14, 16 18,20. Unlike the equation for the $D d 51$ which expresses it in terms of the interacting density of states in the dot, an expression for the $A C$ in terms of the dot Green functions 5253 involves two Green functions $\left(G^{<}\right.$and $\left.G^{r}\right)$ and integration over real time. We find it easier to establish a perturbation expansion for the current itself (rather than for the Green function). To this end we rewrite equation (11) in the interaction representation using the "grand - canonical" Hamiltonian $H_{S B}+\lambda Q_{A}$. The result is, $I_{L(R)}^{T}(t)=-\frac{i e}{\hbar} \sum_{\sigma, k \in L(R)} V_{k d}(t) \operatorname{Tr}_{F_{1}^{A}}\left\{\rho_{F_{1}^{A}}(0) S(-\infty,+\infty) \hat{T}\left[a_{k, \sigma}^{\dagger}(t+0) b^{\dagger}(t) f_{\sigma}(t) S(+\infty,-\infty)\right]\right\}+c . c .$,

where $\hat{T}$ is the usual time - ordering operator, $S(+\infty,-\infty)=\hat{T} e^{-i \int_{-\infty}^{+\infty} H_{T} d t}$ is the usual $\mathrm{S}$ - matrix and $S(-\infty,+\infty)=S^{\dagger}(+\infty,-\infty)$, while $H_{T} \equiv \sum_{k \in L, R ; \sigma}\left(V_{k d}(t) a_{k, \sigma}^{\dagger} c_{d, \sigma}+\right.$ h.c $)$ is the tunneling part of the Hamiltonian $H_{S B}+\lambda Q_{A}$ which is chosen as a perturbation. The operators $a_{k, \sigma}^{\dagger}(t+0), b^{\dagger}(t)$ and $f_{\sigma}(t)$ in the above equation now appear in the interaction representation (this is self evident as indicated by the presence of the $S$ - matrix). We notice that it does not really matter whether one defines the interaction representation 
using the Hamiltonian $H_{S B}+\lambda Q_{A}$ or $H_{S B}$ because $Q_{A}$ commutes with all parts of $H_{S B}$. The factor $S(-\infty,+\infty)$ in equation (12) prevents derivation of Wick's theorem and subsequent development of the Feynman diagrams technique. Indeed, the derivation of Wick's theorem is based on commutation of operators which produces pairings, namely "contractions" (see Ref. 54). Commutation of two operators can not be worked out if one of them is subject to the time - ordering operator while the other one is not. The idea of a closed time

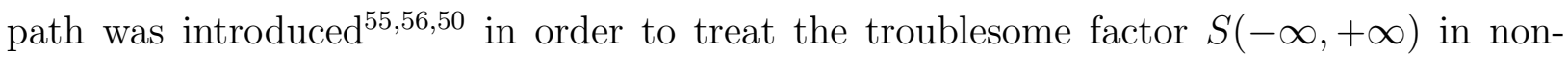
equilibrium systems. The normal time branch is continued and turned back so that this operator becomes a factor within the scope of the time - ordering operator $\left(\hat{T}_{p}\right)$ on the closed time - path. Equation (12), then, can be expressed as,

$$
I_{L(R)}^{T}(t)=-\frac{i e}{\hbar} \sum_{\sigma, k \in L(R)} V_{k d}(t) \operatorname{Tr}_{F_{1}^{A}}\left\{\rho_{F_{1}^{A}}(0) \hat{T}_{p}\left[a_{k, \sigma}^{\dagger}\left(t_{+}\right) b^{\dagger}\left(t_{-}\right) f_{\sigma}\left(t_{-}\right) S_{p}\right]\right\}+c . c .
$$

Here $S_{p}=e^{-i \int_{p} H_{T} d t_{p}}$, where $t_{p}$ is the variable on the closed time - path and $\int_{p}$ means integration over it. The subscript "-" on $t$ signifies that the instant of time is considered on the normal (forward) time branch, while the subscript "+" is used for the backward oriented time branch (see Fig. 2). The choice of the subscripts "+" and "-" for the time arguments of the operators $a_{k, \sigma}^{\dagger}\left(t_{+}\right), b^{\dagger}\left(t_{-}\right)$and $f_{\sigma}\left(t_{-}\right)$is consistent in order to assure that their ordering by the operator $\hat{T}_{p}$ is the same as in Eq. (11). The procedure of transformation from the Heisenberg representation (Eq. (11)) to the closed time path representation (Eq. (13)) is

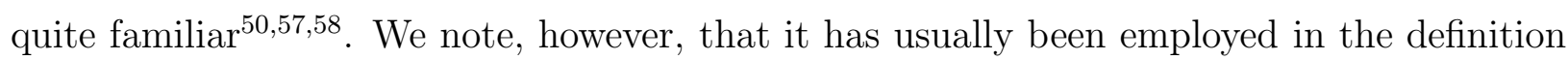
of non-equilibrium Green functions while here it is applied for the current operator.

Yet, perturbation expansion of an expression for the current in the form (13) can not be carried out because of the constraint on traces in the subspace $F_{1}^{A}$. In order to get rid of it we apply the Coleman method $\$ 11$ which is feasible here since the equation (13) appears as an ordinary statistical average of the operator $O_{A} \equiv \hat{T}_{p}\left[a_{k, \sigma}^{\dagger}\left(t_{+}\right) b^{\dagger}\left(t_{-}\right) f_{\sigma}\left(t_{-}\right) S_{p}\right]$ in the subspace $F_{1}^{A}$. Let us choose the initial distribution of the system (before the tunneling was switched on) in the full Hilbert space to be an equilibrium one (grand - canonical ensemble with the "chemical potential" $-\lambda$ ). Then the initial density matrix is, 


$$
\rho(-\infty) \equiv e^{-\beta\left(H_{0}+\lambda Q_{A}\right)} / Z_{G}(\lambda)
$$

where $H_{0} \equiv H_{S B}-H_{T}$ is the unperturbed part of the Hamiltonian (9) and $Z_{G}(\lambda) \equiv$ $\operatorname{Tr}\left\{e^{-\beta\left(H_{0}+\lambda Q_{A}\right)}\right\}$ is the grand - canonical partition function. Now let us consider the expectation values of the operators $O_{A} Q_{A}$ and $Q_{A}$ in the full Hilbert space:

$$
\begin{gathered}
\left\langle O_{A} Q_{A}\right\rangle \equiv \operatorname{Tr}\left\{\rho(0) O_{A} Q_{A}\right\}, \\
\left\langle Q_{A}\right\rangle \equiv \operatorname{Tr}\left\{\rho(0) Q_{A}\right\},
\end{gathered}
$$

where $\rho(0)$ is the density matrix of the system in the full Hilbert space at the zeroth instant of time. When the tunneling is switched on the density matrix evolves in time, so that $\rho(0)$ differs from $\rho(-\infty)$. However, due to the fact that the operator $Q_{A}$ commutes with the Hamiltonian it can still be factorized into separate blocks for each subspace $F_{Q}^{A}$ with different number $Q_{A}$. Thus,

$$
\begin{aligned}
\left\langle O_{A} Q_{A}\right\rangle & =\sum_{Q_{A}=0}^{\infty} \operatorname{Tr}_{F_{Q}^{A}}\left\{\rho_{F_{Q}^{A}}(0) O_{A}\right\} Q_{A} e^{-\beta \lambda Q_{A}} Z_{Q_{A}} / Z_{G}(\lambda) \\
\left\langle Q_{A}\right\rangle & =\sum_{Q_{A}=0}^{\infty} Q_{A} e^{-\beta \lambda Q_{A}} Z_{Q_{A}} / Z_{G}(\lambda)
\end{aligned}
$$

where $\rho_{F_{Q}^{A}}(0)$ is the density matrix for the subspace $F_{Q}^{A}$ at the zeroth instant of time, while $Z_{Q_{A}} \equiv \operatorname{Tr}_{F_{Q}^{A}}\left\{e^{-\beta H_{0}}\right\}$. It is easy to see that in the limit $\lambda \rightarrow \infty$ the ratio of the two expressions written above becomes the expectation value of the operator $O_{A}$ in the physical subspace $F_{1}^{A}$ :

$$
\left\langle O_{A}\right\rangle_{F_{1}^{A}} \equiv \operatorname{Tr}_{F_{1}^{A}}\left\{\rho_{F_{1}^{A}}(0) O_{A}\right\}=\lim _{\lambda \rightarrow \infty} \frac{\left\langle O_{A} Q_{A}\right\rangle}{\left\langle Q_{A}\right\rangle}
$$

It is clear that the operator $O_{A}$ has zero expectation value in the subspace $F_{0}$ because there are neither slave fermions nor slave bosons there. Then $Q_{A}$ can be dropped out of the numerator in (16). This results in our final expression for the current,

$$
I_{L(R)}^{T}(t)=-\frac{i e}{\hbar} \sum_{\sigma, k \in L(R)} V_{k d}(t) \lim _{\lambda \rightarrow \infty} \frac{\left\langle\hat{T}_{p}\left[a_{k, \sigma}^{\dagger}\left(t_{+}\right) b^{\dagger}\left(t_{-}\right) f_{\sigma}\left(t_{-}\right) S_{p}\right]\right\rangle}{\left\langle Q_{A}\right\rangle}+\text { c.c. }
$$

In this equation the averages are taken in the full Hilbert space. It is amenable for standard field - theoretical calculation techniques. 


\section{Diagrammatic expansion of the current}

In the rest of this section we show how to work out a perturbation expansion for the current starting from equation (17) in powers of the tunneling part of the Hamiltonian (9). In principle, a careful calculation of the density matrix $\rho(0)$ is required for the non-equilibrium perturbation expansion. Otherwise, truncation of the full set of diagrams to a finite number might lead to completely wrong results. Indeed, truncation of a perturbation set implies that the effect of the perturbation is small, i.e. higher order terms of the perturbation series are negligible. It is not always ensured by the small value of the perturbation parameter. Consider, for example, an empty dot connected very weakly to a reservoir. The smaller the tunneling between them is, the longer it takes to fill the dot, but eventually the dot becomes full. It means that the occupation of the dot changes by a finite amount although the coupling is infinitesimally small. This "long - times" perturbation problem appears in the energy representation as an infrared divergence 18 . Fortunately, it has been explicitly shown 18 that in the Kondo limit (determined by the conditions (3) and (4a)) the calculation of the Kondo - type contribution to the $D C$ up to sixth order in $V$ can be done using slave boson and slave fermion populations for a disconnected dot. In calculating the $A C$ we also have to impose the condition (4b). The calculation of the denominator in equation (17) is, then, very simple:

$$
\left\langle Q_{A}\right\rangle=n_{b}+2 n_{f} \stackrel{\lambda \rightarrow \infty}{\longrightarrow}\left[1+2 e^{-\beta\left(\epsilon_{d, \sigma}-\mu\right)}\right] e^{-\beta \lambda}
$$

where

$$
\begin{aligned}
& n_{b}=1 /(\exp (\beta \lambda)-1) \text { and } \\
& n_{f}=1 /\left(\exp \left[\beta\left(\epsilon_{d, \sigma}-\mu+\lambda\right)\right]+1\right)
\end{aligned}
$$

are Bose and Fermi functions for the slave particles. We assume here, for simplicity, that the number of spin degrees of freedom $\sigma$ is equal to two.

In order to study the numerator of equation (17) we start from its perturbation expansion in powers of $H_{T}$ (i.e. in powers of $\left.V_{k d}(t)\right)$. Then we transform every term of the expansion 
from the closed time - path to the single time branch (see Ref. 57,59 61). That implies expression of the Green functions as matrices in Keldysh space (see equations (20) below). The next step is a rotation in this space (see Ref. 59,58) resulting in the so called58 "physical representation" for these matrices. Finally, time - translation invariance of the unperturbed Green functions allows us to apply Fourier transform and work in the energy representation.

The diagrams of forth and sixth order in $V_{k d}(t)$ are shown in Fig. 3. Recall that disconnected diagrams need not be considered in the Schwinger - Keldysh formalism. Contribution of a disconnected diagram can be factorized into contributions of the connected and disconnected parts. Summation over all possible disconnected parts yields $\left\langle\hat{T}_{p} S_{p}\right\rangle$ that is $\langle S(-\infty,-\infty)\rangle$ which is equal to unity. Notice, that diagram "h" is a crossed diagram. It is not included in the ever used non - crossing approximation. We now formulate the basic rules for drawing the diagrams and writing down the corresponding analytical expressions.

1. In order to obtain a diagram of the $m^{\text {th }}$ order in $V$, draw a circle (it appears as a polygon in the figures) consisting of $m$ alternating slave - boson and slave - fermion lines going in the same direction. The number $m$ has to be even because every tunneling vertex contains only one slave - boson and one slave - fermion operator. The diagram must include only one such circle, otherwise it would produce contribution to the current of first or higher order in powers of $\exp (-\beta \lambda)$ (see Ref. 41) that vanishes at $\lambda \rightarrow \infty$. We represent the diagrams by closed circles rather than by open lines as it is usually done since the external operators $b^{\dagger}$ and $f_{\sigma}$ in equation (17) are taken at the same time $t_{-}$. Connect the vertices by lead - electron lines. Remember that only the two types of vertices shown in Fig. 1 are allowed.

2. Introduce the following $2 \times 2$ (Keldysh) matrices for every lead - electron, slave fermion and slave - boson lines respectively:

$$
g_{k \sigma}(\epsilon) \equiv\left(\begin{array}{cc}
0 & g_{k \sigma}^{a}(\epsilon) \\
g_{k \sigma}^{r}(\epsilon) & g_{k \sigma}^{c}(\epsilon)
\end{array}\right), \quad \xi_{\sigma}(\omega) \equiv\left(\begin{array}{cc}
0 & \xi_{\sigma}^{a}(\omega) \\
\xi_{\sigma}^{r}(\omega) & \xi_{\sigma}^{c}(\omega)
\end{array}\right)
$$




$$
d(\nu) \equiv\left(\begin{array}{cc}
0 & d^{a}(\nu) \\
d^{r}(\nu) & d^{c}(\nu)
\end{array}\right) .
$$

The indices "r", "a" and "c" stand to denote retarded, advanced and correlation Green functions. They are defined explicitly as follows:

$$
\begin{aligned}
g_{k \sigma}^{r(a)}(\epsilon) & =\frac{1}{\epsilon-\epsilon_{k}+\mu \pm i \gamma} \\
g_{k \sigma}^{c}(\epsilon) & =\left[1-2 f\left(\epsilon_{k}\right)\right]\left[g_{k \sigma}^{r}(\epsilon)-g_{k \sigma}^{a}(\epsilon)\right] \\
\xi_{\sigma}^{r(a)}(\omega) & =\frac{1}{\omega-\epsilon_{d, \sigma}+\mu-\lambda \pm i \gamma} \\
\xi_{\sigma}^{c}(\omega) & =\left[1-2 \exp \left(-\beta\left(\epsilon_{d, \sigma}-\mu\right)\right) \exp (-\beta \lambda)\right]\left[\xi_{\sigma}^{r}(\omega)-\xi_{\sigma}^{a}(\omega)\right] \\
d^{r(a)}(\nu) & =\frac{1}{\nu-\lambda \pm i \gamma} \\
d^{c}(\nu) & =[1-2 \exp (-\beta \lambda)]\left[d^{r}(\nu)-d^{a}(\nu)\right],
\end{aligned}
$$

where $f\left(\epsilon_{k}\right)=1 /\left(\exp \left[\beta\left(\epsilon_{k}-\mu\right)\right]+1\right)$ is the Fermi function for lead electrons, the factors $\exp \left[-\beta\left(\epsilon_{d, \sigma}-\mu\right)\right] \exp (-\beta \lambda)$ and $\exp (-\beta \lambda)$ are the limiting forms of the Fermi and the Bose functions (19) respectively at large $\lambda$. We note that different representations can be used for the matrices (20) (see Ref. 58 61). Here we employ the so called 58 "physical representation".

3. Introduce the following tensors for every internal vertex (represented by a closed circle in Fig. 3). For the vertex drawn in Fig. 因a:

$$
\tilde{V}_{k d} J_{s}\left(W_{(k)} / \Omega\right) e^{i s \alpha_{(k)}} \eta_{i j}^{m}
$$

For the vertex drawn in Fig. ㅂb:

$$
\tilde{V}_{k d}^{*} J_{q}\left(W_{(k)} / \Omega\right) e^{-i q \alpha_{(k)}} \eta_{i j}^{m},
$$

where $J_{s}$ and $J_{q}$ are Bessel functions. The index " $(k)$ " reminds us that, despite the fact that $W$ and $\alpha$ do not depend on $k$, they depend on the lead to which $k$ belongs. The 
factors $J_{s(q)}\left(W_{(k)} / \Omega\right) \exp \left( \pm i s(q) \alpha_{(k)}\right)$ originate from the time - dependence of $V_{k d}(t)$. Indeed, substituting (2) into (7) we find

$$
\begin{aligned}
V_{k d}(t) & =\tilde{V}_{k d} e^{\frac{i}{\hbar}\left[\phi_{(k)} t+i W_{(k)} / \Omega \sin \left(\Omega t+\alpha_{(k)}\right)-\beta_{(k)}\right]} \\
& =\tilde{V}_{k d} \sum_{s=-\infty}^{\infty} J_{s}\left(W_{(k)} / \Omega\right) e^{\frac{i}{\hbar}\left[\phi_{(k)} t+s \Omega t+s \alpha_{(k)}-\beta_{(k)}\right]}
\end{aligned}
$$

The time - dependent factors appearing in this expression will surface when we impose energy conservation (see below), the phases $\beta_{(k)}$ cancel, while the rest enters equations (22) and (23). The physical meaning of $s$ is the number of photons emitted when an electron goes from the dot to a lead, while $q$ is the number of photons absorbed by an electron going from a lead to the dot. Both $s$ and $q$ can assume negative values. The tensor $\eta$ is given by the following expressions:

$$
\eta_{i j}^{1}=\frac{1}{\sqrt{2}}\left(\begin{array}{ll}
1 & 0 \\
0 & 1
\end{array}\right), \quad \eta_{i j}^{2}=\frac{1}{\sqrt{2}}\left(\begin{array}{cc}
0 & 1 \\
1 & 0
\end{array}\right) .
$$

It appears as a result of the transformation leading from from time integration over past-ward going time branch to integration over normal time axis which requires a change of sign. The above form is consistent with the physical representation for the Keldysh matrices and tensors 50 .

For the external vertex (represented by an open circle in Fig. 3) containing the operators $a_{k, \sigma}^{\dagger}\left(t_{+}\right), b^{\dagger}\left(t_{-}\right)$and $f_{\sigma}\left(t_{-}\right)$of equation (17) write the factor $J_{s}\left(W_{L} / \Omega\right) \exp \left(i s \alpha_{L}\right)$ as for the internal vertices but do not insert the tensor $\eta$. Instead, close around the product of matrices and tensors introduced above by the row $2^{-1 / 2}(1,1)$ at the $\xi$ matrix and the vectors $\frac{1}{\sqrt{2}}\left(\begin{array}{c}-1 \\ 1\end{array}\right)$ and $\frac{1}{\sqrt{2}}\left(\begin{array}{l}1 \\ 1\end{array}\right)$ at the matrices $g$ and $d$ respectively as we show in the example (equation (28)) below. The external vertex differs from the internal ones because the time - variable for every external operator $a_{k, \sigma}^{\dagger}\left(t_{+}\right), b^{\dagger}\left(t_{-}\right)$or $f_{\sigma}\left(t_{-}\right)$is chosen on a certain $(+$or -$)$branch and these time branches are different for $a_{k, \sigma}^{\dagger}, b^{\dagger}$ and $f_{\sigma}$. In an internal vertex, the time - variables for all the operators are chosen on a single time branch following by summation over the two time branches. 
4. Conserve spin in every vertex. As for energy, it should be conserved in every internal vertex taking into account emission (absorption) of $s(q)$ photons and the different values assumed by the static potential energy $\phi_{(k)}$ on the left and right lead. The energy in the external vertex is not conserved. The pertinent energy difference is equal to the frequency of the current. Therefore, introduce for this vertex the factor $\delta_{n,\left(q-s+q_{1}-s_{1}+\ldots\right)}$, where $n$ has the meaning of the total number of absorbed photons. Summation over $n$ will be carried out in equation (27) below.

5. Sum over energy, momentum and spin. Do not specify to which lead the momenta belong. It will be taken care of later on.

6. Sum over numbers of photons $q, s, q_{1}, \ldots$

7. Multiply the result by the factor

$$
\frac{-i e}{\hbar\left\langle Q_{A}\right\rangle}(-i)^{m-1}(i)^{3 m / 2}(-1)^{F}(2 \pi)^{-m / 2}
$$

where $F$ is the number of closed electron - fermion loops. The first factor in the above expression comes from equation (17), the second one is implied by the $m-1$ order of the expansion of the $S$ - matrix. As for the next two factors, recall that an application of the Wick's theorem results in pairings of creation and annihilation operators which are, then, expressed through Green functions. The last factor simply results from the Fourier transform.

8. At this stage take the limit $\lambda \rightarrow \infty$.

9. Let us denote the expression obtained through the rules that have been listed by the symbol $\Upsilon_{A}(n)$. Then the current is given by the following equation:

$$
\begin{aligned}
& I_{L(R)}^{T}(t)=\frac{1}{\pi} \sum_{n} \sum_{\left(k_{1}\right),\left(k_{2}\right), \ldots=L, R}\left\{\operatorname{Re}\left[\Upsilon_{A}(n)\right] \cos \left(n \Omega t-\alpha_{(k),\left(k_{1}\right), \ldots}\right)\right. \\
& \left.+\operatorname{Im}\left[\Upsilon_{A}(n)\right] \sin \left(n \Omega t-\alpha_{\left.(k),\left(k_{1}\right), \ldots\right)}\right)\right\},
\end{aligned}
$$


where $\alpha_{(k),\left(k_{1}\right), \ldots} \equiv(s-q) \alpha_{L}+\left(s_{1}-q_{1}\right) \alpha_{\left(k_{1}\right)}+\left(s_{2}-q_{2}\right) \alpha_{\left(k_{2}\right)}+\ldots$, while $\sum_{\left(k_{1}\right),\left(k_{2}\right), \ldots=L, R}$ means summation over the leads to which $k_{1}, k_{2}$, etc. belong. There is no summation over $(k)$. The electron line coming out of the external vertex has momentum $k$ belonging only to the left (right) lead since we are calculating current through the left (right) barrier. The other momenta run through both leads.

As an example we write below the expression which is obtained using the rules listed above for the diagram drawn in Fig. 3e:

$$
\begin{aligned}
\Upsilon_{A}(n)= & \lim _{\lambda \rightarrow \infty} \frac{-i e}{\hbar\left\langle Q_{A}\right\rangle} \frac{1}{16} \frac{1}{(2 \pi)^{3}} \sum_{q, q_{1}, q_{2}, s, s_{1}, s_{2}} \delta_{n,\left(q+q_{1}+q_{2}-s-s_{1}-s_{2}\right)} \\
& \cdot J_{q}\left(\frac{W_{L}}{\Omega}\right) J_{s}\left(\frac{W_{L}}{\Omega}\right) J_{q_{1}}\left(\frac{W_{\left(k_{1}\right)}}{\Omega}\right) J_{s_{1}}\left(\frac{W_{\left(k_{1}\right)}}{\Omega}\right) J_{q_{2}}\left(\frac{W_{\left(k_{2}\right)}}{\Omega}\right) J_{s_{2}}\left(\frac{W_{\left(k_{2}\right)}}{\Omega}\right) \\
& \cdot \sum_{k_{1} \in L ; k, k_{2} \in L, R ; \sigma, \sigma_{2}}\left|\tilde{V}_{k d}\right|^{2}\left|\tilde{V}_{k_{1} d}\right|^{2}\left|\tilde{V}_{k_{2} d}\right|^{2} \iiint \int d \omega_{1} d \omega_{2} d \nu_{1} d \nu_{2} \\
& \cdot \sum_{i, j, m, \ldots, i_{5}, j_{5}, m_{5}}(1,1)_{i} \xi^{i i_{1}}\left(\omega_{1}\right) \eta_{i_{1} j_{1}}^{m_{1}} g_{k_{1} \sigma}^{j_{1} j_{2}}\left(\omega_{1}-\nu-\phi_{\left(k_{1}\right)}-q_{1} \Omega\right) d^{m_{1} m_{2}}(\nu) \eta_{i_{2} j_{2}}^{m_{2}} \\
& \cdot \xi^{i_{1} i_{2}}\left(\omega_{1}-\left(q_{1}-s_{1}\right) \Omega\right) \eta_{i_{3} j_{3}}^{m_{3}} g_{k \sigma}^{j_{3} j}\left(\omega_{1}-\nu_{1}-\phi_{L}-\left(q+q_{1}+q_{2}-s_{1}-s_{2}\right) \Omega\right) \\
& \cdot d^{m_{3} m_{4}}\left(\nu_{1}+\left(q_{2}-s_{2}\right) \Omega\right) \eta_{i_{4} j_{4}}^{m_{4}} \xi^{i_{4} i_{5}}\left(\omega_{2}\right) \eta_{i_{5} j_{5}}^{m_{5}} g_{k_{2} \sigma_{2}}^{j_{5} j_{4}}\left(\omega_{2}-\nu_{1}-\phi_{\left(k_{2}\right)}-q_{2} \Omega\right) \\
& \cdot d^{m_{5} m}\left(\nu_{1}\right)\left(\begin{array}{c}
-1 \\
1 \\
1
\end{array}\right)_{j} .
\end{aligned}
$$

In the above equation the tensor product is to be expanded followed by integration over internal variables $\omega_{1}, \omega_{2}, \nu_{1}$ and $\nu_{2}$ is to be carried out. Using the Mathematica program we have carried it out for the above equation and for the analogous one given by the diagram drawn in Fig. 3f. Whereas the calculation of the current could not be completed (see below) it was possible to inspect the emergence of a Kondo behavior through the appearance of a term $\sum_{p} \frac{f(p)}{\epsilon_{k}-\epsilon_{p}}$ where $f(p)=f\left(\epsilon_{p}\right)$ is the Fermi function for lead electrons of quantum number $p$. It is well known that this term is characteristic for the Kondo effect (above the Kondo temperature). Accordingly, the resulting expression for diagram $3 \mathrm{f}$ does not exhibit a Kondo - type behavior (i.e. $\ln T$ - divergence in the linear response) while the one obtained for diagram 3e and represented by equation (28) does. The equations in their final form are 
very long and cumbersome and will not be shown here. Moreover, expressions appearing in the intermediate stages are unusually long and often overflowing the memory of a typical present - day workstation. We stress that this occurs only when computing the $A C$ (calculations appropriate for the $D C$ are much simpler). These manipulations pertaining to $A C$ appear to be especially cumbersome for the crossed diagram $3 \mathrm{~h}$. We argue that, physically, the main source of complications results from the admixture of the Kondo resonance with usual resonant tunneling which is present in the Anderson model. Although the latter contribution is exponentially small within the relevant range of parameters, governed by terms like $\exp \left(\epsilon_{d, \sigma} /(k T)\right)$ and may frequently be neglected in the Kondo regime, its omission can not be assumed in this calculation scheme before arriving at the final stages. We are therefore content by having introduced the systematic calculation scheme for the general time dependent Anderson model, and exposing some diagrams that manifest a Kondo behavior. With rapid improvement of workstation capacities and analytic software programs the evaluation of all diagrams of sixth order presented here can be completed according to the rules specified above. With this final note we now move on to complete the calculation of the current within an effective theory which still captures most of the pertinent subtle physics.

\section{TIME - DEPENDENT SCHRIEFFER - WOLFF TRANSFORMATION}

In this section we transform the time - dependent Anderson Hamiltonian to a time dependent Kondo Hamiltonian. We use a procedure similar to the one proposed by Schrieffer

and Wolff4 for the time - independent Anderson model but extend it to time - dependent problems.

As we did in the previous section we start from the canonical transformation (5) of the Anderson Hamiltonian (11) whose purpose is to transfer the dependence on time into the perturbation part. It results in the Hamiltonian $H_{A}$ (Eq. (的)). Then we further transform this Hamiltonian as,

$$
H_{A}^{\prime}=e^{S} H_{A} e^{-S}-i e^{S} \frac{\partial}{\partial t} e^{-S}
$$


where the operator $S$ is defined according to a specific prescription. In the spirit of Ref. 44 we expand the right hand side of Eq. (29) in powers of $S$. We notice that $S$ does not commute with $\frac{\partial S}{\partial t}$ so this manipulation should be done with care. Then we require the operator $S$ to satisfy the equation,

$$
i \frac{\partial S}{\partial t}+H_{T}+\left[S, H_{0}\right]=0
$$

Here $H_{T} \equiv \sum_{k \in L, R ; \sigma}\left(V_{k d}(t) a_{k, \sigma}^{\dagger} c_{d, \sigma}+\right.$ h.c $)$ is the tunneling part of the Hamiltonian $H_{A}$ and $H_{0} \equiv \sum_{k \in L, R ; \sigma} \epsilon_{k} a_{k, \sigma}^{\dagger} a_{k, \sigma}+\sum_{\sigma} \epsilon_{d, \sigma} c_{d, \sigma}^{\dagger} c_{d, \sigma}+\frac{1}{2} U \sum_{\sigma, \sigma^{\prime} \neq \sigma} n_{d, \sigma} n_{d, \sigma^{\prime}}$ is the rest of it. If $S$ is proportional to $V$ (which is indeed the case as we show at the end of this section) then solving Eq. (30) eliminates from the Hamiltonian $H_{A}^{\prime}$ terms which are of first order in $V$, leaving only terms of higher orders which are naturally smaller. Upon collecting terms of zero and second order in $V$ the Hamiltonian $H_{A}^{\prime}$ can be written in the following form:

$$
H_{A}^{\prime}=H_{0}+\frac{1}{2}\left[S, H_{T}\right]
$$

Following Ref.44 we omit terms of $3 \mathrm{~d}$ and higher orders in $V$. Our transformation differs from that of Ref. 4 due to the presence of the term $-i e^{S} \frac{\partial}{\partial t} e^{-S}$ in Eq. (29) and, correspondingly, the term $i \frac{\partial S}{\partial t}$ in Eq. (30).

Now we turn to the task of solving Eq. (30), and look for a solution of the form,

$$
S=S_{1}-S_{1}^{\dagger}
$$

The operator $S_{1}$, then, should satisfy the equation

$$
i \frac{\partial S_{1}}{\partial t}+H_{T}^{\text {out }}+\left[S_{1}, H_{0}\right]=0
$$

where $H_{T}^{\text {out }} \equiv \sum_{k \in L, R ; \sigma} V_{k d}(t) a_{k, \sigma}^{\dagger} c_{d, \sigma}$ is the part of the Hamiltonian responsible for tunneling out of the dot. The operator $S$ is anti - hermitian which assures the hermiticity of $H_{A}^{\prime}$.

We are now looking for a solution of equation (33) in the form,

$$
S_{1}=\sum_{k \in L, R ; \sigma} A_{k, \sigma}(t)\left[\frac{n_{d,-\sigma}}{\epsilon_{k}-\epsilon_{d, \sigma}-U}+\frac{\left(1-n_{d,-\sigma}\right)}{\epsilon_{k}-\epsilon_{d, \sigma}}\right] a_{k, \sigma}^{\dagger} c_{d, \sigma} .
$$


The sum appearing in the square brackets is the inverse of the operator $\zeta_{k, \sigma} \equiv \epsilon_{k}-\epsilon_{d, \sigma}-$ $U n_{d,-\sigma}$. Schrieffer and Wolff found $A_{k, \sigma}(t)=\tilde{V}_{k d}$, where $\tilde{V}_{k d}$ is, of course, time - independent.

Substituting expression (34) into equation (33) we find that $A_{k, \sigma}(t)$ must satisfy the following equation:

$$
i \dot{A}_{k}(t) \zeta_{k, \sigma}^{-1}-A_{k, \sigma}(t)+V_{k d}(t)=0
$$

A proper solution of this equation is

$$
A_{k, \sigma}(t)=\mathcal{V}_{k d} \sum_{s=-\infty}^{\infty} J_{s}\left(W_{(k)} / \Omega\right) e^{i\left(\phi_{(k)}+s \Omega\right) t+i s \alpha_{(k)}} \frac{\zeta_{k, \sigma}}{\phi_{(k)}+s \Omega+\zeta_{k, \sigma}}
$$

where $\mathcal{V}_{k d} \equiv \tilde{V}_{k d} \exp \left[-i\left(W_{(k)} / \Omega\right) \sin \alpha_{(k)}\right]$. Recall that the symbol $(k)$ means "L" or "R" depending on whether $k$ belongs to the left or to the right lead, while $J_{s}\left(\frac{W}{\Omega}\right)$ are Bessel's functions. The general solution of Eq. (35) contains also a term: $\propto e^{-i \zeta_{k, \sigma} t}$, but the requirement that $A_{k, \sigma}(t)$ should be time - independent in the absence of external potentials enforces the prefactor to vanish.

Employing now equations (31), (32), (34) and (36) we obtain the desired form of the Hamiltonian, in exact correspondence with Ref. 4 ,

$$
\begin{aligned}
H_{A}^{\prime}= & H_{0}+\sum_{k, k^{\prime} \in L, R ; \sigma}\left(W_{k^{\prime} k}(t)-1 / 2 J_{k^{\prime} k}(t) n_{d,-\sigma}\right) a_{k^{\prime}, \sigma}^{\dagger} a_{k, \sigma} \\
& +1 / 2 \sum_{k, k^{\prime} \in L, R ; \sigma} J_{k^{\prime} k}(t) a_{k^{\prime},-\sigma}^{\dagger} a_{k, \sigma} c_{d, \sigma}^{\dagger} c_{d,-\sigma} \\
& -\sum_{k \in L, R ; \sigma}\left(W_{k k}(t)-1 / 2 J_{k k}(t) n_{d,-\sigma}\right) c_{d, \sigma}^{\dagger} c_{d, \sigma} \\
& -1 / 4 \sum_{k, k^{\prime} \in L, R ; \sigma}\left[J_{k^{\prime} k}(t) a_{k^{\prime},-\sigma}^{\dagger} a_{k, \sigma}^{\dagger} c_{d, \sigma} c_{d,-\sigma}+\text { h.c. }\right],
\end{aligned}
$$

where

$$
\begin{aligned}
J_{k^{\prime} k}(t)= & \mathcal{V}_{k^{\prime} d} \mathcal{V}_{k d}^{*} \exp \left[i\left(\phi_{\left(k^{\prime}\right)}-\phi_{(k)}\right) t\right] . \\
& \cdot \sum_{s^{\prime}, s=-\infty}^{+\infty} J_{s^{\prime}}\left(\frac{W_{\left(k^{\prime}\right)}}{\Omega}\right) J_{s}\left(\frac{W_{(k)}}{\Omega}\right) \exp \left[i\left(s^{\prime}-s\right) \Omega t+i\left(s^{\prime} \alpha_{\left(k^{\prime}\right)}-s \alpha_{(k)}\right)\right] . \\
& \cdot\left(\frac{1}{\epsilon_{k^{\prime}}+\phi_{\left(k^{\prime}\right)}+s^{\prime} \Omega-\epsilon_{d, \sigma}}+\frac{1}{\epsilon_{k}+\phi_{(k)}+s \Omega-\epsilon_{d, \sigma}}+\right. \\
& \left.+\frac{1}{\epsilon_{k^{\prime}}+\phi_{\left(k^{\prime}\right)}+s^{\prime} \Omega-\epsilon_{d, \sigma}-U}+\frac{1}{\epsilon_{k}+\phi_{(k)}+s \Omega-\epsilon_{d, \sigma}-U}\right),
\end{aligned}
$$




$$
\begin{aligned}
W_{k^{\prime} k}(t)= & \frac{1}{2} \mathcal{V}_{k^{\prime} d} \mathcal{V}_{k d}^{*} \exp \left[i\left(\phi_{\left(k^{\prime}\right)}-\phi_{(k)}\right) t\right] . \\
& \cdot \sum_{s^{\prime}, s=-\infty}^{+\infty} J_{s^{\prime}}\left(\frac{W_{\left(k^{\prime}\right)}}{\Omega}\right) J_{s}\left(\frac{W_{(k)}}{\Omega}\right) \exp \left[i\left(s^{\prime}-s\right) \Omega t+i\left(s^{\prime} \alpha_{\left(k^{\prime}\right)}-s \alpha_{(k)}\right)\right] . \\
& \cdot\left(\frac{1}{\epsilon_{k^{\prime}}+\phi_{\left(k^{\prime}\right)}+s^{\prime} \Omega-\epsilon_{d, \sigma}}+\frac{1}{\epsilon_{k}+\phi_{(k)}+s \Omega-\epsilon_{d, \sigma}}\right) .
\end{aligned}
$$

The form of this Hamiltonian is the same as that of Ref. 4 but the matrix elements $J_{k^{\prime} k}(t)$ and $W_{k^{\prime} k}(t)$ are evidently distinct.

In the Kondo regime, the important subspace $F_{1}^{K}$ of the full Hilbert space is that one for which the dot is occupied by one electron. The last term in the Hamiltonian (37) is not relevant for this subspace. The third term in equation (37) can be absorbed into the definitions of $\epsilon_{d, \sigma}$ and $U$. Besides, we have $n_{d, \sigma} n_{d,-\sigma}=0$ and $\sum_{\sigma} n_{d, \sigma}=1$ in the subspace $F_{1}^{K}$, so that the Coulomb interaction term (present in $H_{0}$ ) vanishes and the one - particle energy term for the dot becomes a $c$ - number. The remaining terms represent the Kondo (also called "s - d") Hamiltonian plus a potential scattering term,

$$
\begin{aligned}
H_{K}= & \sum_{k \in L, R ; \sigma} \epsilon_{k} a_{k, \sigma}^{\dagger} a_{k, \sigma}+\sum_{k, k^{\prime} \in L, R ; \sigma}\left(W_{k^{\prime} k}(t)-1 / 2 J_{k^{\prime} k}(t) n_{d,-\sigma}\right) a_{k^{\prime}, \sigma}^{\dagger} a_{k, \sigma}+ \\
& +1 / 2 \sum_{k, k^{\prime} \in L, R ; \sigma} J_{k^{\prime} k}(t) a_{k^{\prime},-\sigma}^{\dagger} a_{k, \sigma} c_{d, \sigma}^{\dagger} c_{d,-\sigma} .
\end{aligned}
$$

Two comments are in order here: (i) Any procedure toward calculation of physical quantities should take into account the fact that, out of the full Hilbert space, the system is projected onto a subspace $F_{1}^{K}$ in which the dot is occupied by one (and only one) electron. (ii) At this stage one might be tempted to express the electron creation - annihilation operators in the dot through spin operators, thus arriving at the familiar form时 4 of the Kondo Hamiltonian. But then one would realize that the spin operators do not obey the usual commutation rules. In order to overcome this obstacle, fictitious (auxiliary) fermions might be introduced 42 . But this leads one back to equation (39). In other words, auxiliary fermions which are sometimes regarded as artificial particles introduced to represent spins are real electrons in the dot (impurity atom) subject to the constraint specified in (i).

Calculation of the tunneling current starting from the Kondo Hamiltonian (39) is possible for arbitrary field strengths and frequency provided the inequalities (4) are satisfied. Yet, 
inspecting a typical experimental setup垷 one may consider somewhat weaker external fields and lower frequencies, so that

$$
\left|\phi_{L(R)}\right|, \Omega, W_{L}, W_{R} \ll\left|\epsilon_{d, \sigma}\right|, \epsilon_{d, \sigma}+U \text {. }
$$

Expressions (38) for $J_{k^{\prime} k}(t)$ and $W_{k^{\prime} k}(t)$ then significantly simplify. Indeed, at small $W / \Omega$ the Bessel function $J_{s}(W / \Omega)$ rapidly decreases with increasing $s$. At large $W / \Omega$ it decays strongly once $s$ exceeds $W / \Omega$. Therefore we can restrict $s \Omega$ to be less than or of the order of $\max (\Omega, W)$, that is, $s \Omega \ll\left|\epsilon_{d, \sigma}\right|, \epsilon_{d, \sigma}+U$. In the formation of the Kondo resonance the most important states are those with energies $\left|\epsilon_{k}\right| \ll\left|\epsilon_{d, \sigma}\right|, \epsilon_{d, \sigma}+U$ (see Ref. 44). Then $\left|\zeta_{k, \sigma}\right| \approx\left|\epsilon_{d, \sigma}\right|$, if $n_{d,-\sigma}=0$, or $\left|\zeta_{k, \sigma}\right| \approx \epsilon_{d, \sigma}+U$, if $n_{d,-\sigma}=1$ (see definition after Eq. 34, and recall that $n_{d,-\sigma}$ is discrete). Therefore the conditions (40) assure that

$$
\phi_{(k)}+s \Omega \ll\left|\zeta_{k, \sigma}\right|
$$

(recall that $\phi_{(k)}$ refers to $\phi_{L}$ or $\left.\phi_{R}\right)$. Therefore we can neglect the term $\phi_{(k)}+s \Omega$ in the denominator of the expression (36). Its right hand side is greatly simplified, and now becomes,

$$
A_{k, \sigma}(t) \approx V_{k d}(t)
$$

where $V_{k d}(t)$ is defined by Eq. (17). Expressions for the matrix elements $J_{k^{\prime} k}$ and $W_{k^{\prime} k}(t)$ simplify as well. First, they become time - independent if $k$ and $k^{\prime}$ belong to the same lead. Moreover, they do not depend on potential shifts of each lead separately but only on their difference, $\Delta_{L}-\Delta_{R}$. For a monochromatic potential difference between the leads we then define

$$
\Delta_{L R} \equiv \Delta_{L}-\Delta_{R} \equiv \phi^{d c}+W \cos (\Omega t+\alpha)
$$

Then the matrix elements $J_{k^{\prime} k}(t)$ and $W_{k^{\prime} k}(t)$ can be expressed in quite a simple form:

$$
\begin{gathered}
J_{k^{\prime} k}(t)= \begin{cases}\tilde{J}_{k^{\prime} k} \exp \left[\frac{i}{\hbar} \int_{-\infty}^{t} d t_{1} \Delta_{L R}\left(t_{1}\right)\right], & \text { if } k^{\prime} \in L, k \in R \\
\tilde{J}_{k^{\prime} k}, & \text { if } k^{\prime}, k \in L \text { or } k^{\prime}, k \in R\end{cases} \\
W_{k^{\prime} k}(t)=\left\{\begin{array}{ll}
\tilde{W}_{k^{\prime} k} \exp \left[\frac{i}{\hbar} \int_{-\infty}^{t} d t_{1} \Delta_{L R}\left(t_{1}\right)\right], & \text { if } k^{\prime} \in L, k \in R \\
\tilde{W}_{k^{\prime} k}, & \text { if } k^{\prime}, k \in L \text { or } k^{\prime}, k \in R
\end{array},\right.
\end{gathered}
$$


where

$$
\begin{aligned}
\tilde{J}_{k^{\prime} k} & \equiv \tilde{V}_{k^{\prime} d} \tilde{V}_{k d}^{*}\left(\frac{1}{\epsilon_{k}-\epsilon_{d, \sigma}}+\frac{1}{\epsilon_{k^{\prime}}-\epsilon_{d, \sigma}}-\frac{1}{\epsilon_{k}-\epsilon_{d, \sigma}-U}-\frac{1}{\epsilon_{k^{\prime}}-\epsilon_{d, \sigma}-U}\right) \\
\tilde{W}_{k^{\prime} k} & \equiv \frac{1}{2} \tilde{V}_{k^{\prime} d} \tilde{V}_{k d}^{*}\left(\frac{1}{\epsilon_{k}-\epsilon_{d, \sigma}}+\frac{1}{\epsilon_{k^{\prime}}-\epsilon_{d, \sigma}}\right)
\end{aligned}
$$

are time - independent. The matrix elements $J_{k^{\prime} k}(t)$ and $W_{k^{\prime} k}(t)$ for $k^{\prime} \in R, k \in L$ satisfy the hermiticity relations $J_{k k^{\prime}}(t)=J_{k^{\prime} k}^{*}(t)$ and $W_{k k^{\prime}}(t)=W_{k^{\prime} k}^{*}(t)$. We note that at small external fields (40) the transformation (determined by Eqs. (34) and (42)) and the form of the matrix elements $J_{k^{\prime} k}(t)$ and $W_{k^{\prime} k}(t)$ are very similar to those of Ref. 4 although they are still different due to the time - dependence of $V_{k d}(t)$. We further note that the Hamiltonian (39) with the coupling constants (44) can alternatively be obtained by application of the canonical transformation (5) to the usual Kondo Hamiltonian with time - dependence added only to the leads (while the coupling of conduction electrons to the impurity (dot) remains time - independent). This is true, however, only at small external fields (40). At stronger fields equations (38) must be used for computing the matrix elements $J_{k^{\prime} k}(t)$ and $W_{k^{\prime} k}(t)$.

In order to have a more compact form of the Hamiltonian we hereafter imply the limit of infinite $U$. It is obvious from equations (44) and (45) that this choice does not lead to any qualitative changes. Indeed, it just slightly affects the values of $J_{k^{\prime} k}$. The main advantage of this choice is that at $U=\infty$ the equality, $W_{k^{\prime} k}=1 / 2 J_{k^{\prime} k}$ holds, which eliminates one more parameter. Furthermore, in the subspace $F_{1}^{K}$ the following identity holds, namely, $1-n_{d,-\sigma}=n_{d, \sigma}$. Then $H_{K}$ can be expressed as

$$
\begin{aligned}
H_{K} & =\sum_{k \in L, R ; \sigma} \epsilon_{k} a_{k, \sigma}^{\dagger} a_{k, \sigma}+ \\
& +1 / 2 \sum_{k, k^{\prime} \in L, R ; \sigma} J_{k^{\prime} k}(t)\left[a_{k^{\prime},-\sigma}^{\dagger} a_{k, \sigma} c_{d, \sigma}^{\dagger} c_{d,-\sigma}+a_{k^{\prime}, \sigma}^{\dagger} a_{k, \sigma} c_{d, \sigma}^{\dagger} c_{d, \sigma}\right] .
\end{aligned}
$$

There is only one coupling constant $J_{k^{\prime} k}(t)$ in this expression which is equal for both coupling terms. Equation (46) constitutes our final form of the time - dependent Hamiltonian which we use in the next section to obtain the tunneling current.

In concluding this section we would like to point out that the main idea of the Schrieffer Wolff transformation is based on a projection of the system out of the full Hilbert space onto 
the subspace $F_{1}^{K}$ for which the dot is occupied by one (and only one) electron. Unoccupied and doubly - occupied subspaces are forbidden. At first glance it looks as a small reduction of space dimension. Yet, as we show in the next section it greatly simplifies the calculation of the tunneling current for time - dependent problems. The main physical reason seems to be the fact that by fixing the number of electrons in the dot one separates the Kondo resonance from the usual resonant tunneling. The latter is exponentially small in the Kondo regime but it is formally present in the Anderson model. Admixture of two different physical processes within the same calculation scheme seems to be the main source of complication. Another advantage of the Kondo Hamiltonian (39) (or (46)) is readily seen from the expressions (39), (43), (44) and (45). Namely, the number of independent parameters is significantly reduced. Indeed, instead of six parameters controlling the external fields in the Anderson model $\left(\phi_{L(R)}, W_{L(R)}, \Omega\right.$ and the phase difference $\left.\alpha_{L}-\alpha_{R}\right)$ we are left with only three: $\phi^{d c}$, $W$ and $\Omega$. Instead of three independent internal parameters $V_{k d}, \epsilon_{d, \sigma}$ and $U$ there are only two important combinations $J_{k^{\prime} k}$ and $W_{k^{\prime} k}$. Moreover, it becomes obvious that the limit of infinite $U$ does not imply any qualitative changes in the results. It allows us to get rid of one more parameter.

\section{PERTURBATION EXPANSION OF THE CURRENT IN THE KONDO MODEL}

\section{A. Expression for the tunneling current}

In this section we define the tunneling current using the time - dependent Kondo Hamiltonian (46) and then develop a non-equilibrium technique to expand it in powers of the coupling strength $J_{k^{\prime} k}$. Although the details of calculation are substantially distinct from those used in section [1], the basic algorithm is quite similar. Of course, we do not need to introduce slave particles here, because the unperturbed part is bilinear in creation - annihilation operators and there is no obstacle in carrying out perturbation expansion in powers of 
the interaction which contains a small parameter $J_{k^{\prime} k}$. The starting point is, again, defining the current using commutation of the Hamiltonian (46) with the number - of - particles operator. It yields, in the Heisenberg representation,

$$
\begin{aligned}
I(t)=-\frac{i e}{\hbar}\left\langle\left[H_{K}, N_{L}\right]\right\rangle=\frac{i e}{\hbar}\left\langle\left[H_{K}, N_{R}\right]\right\rangle= \\
=\frac{e}{\hbar} \sum_{k^{\prime} \in L, k \in R ; \sigma} \operatorname{Im}\left\{J_{k^{\prime} k}(t) \operatorname{Tr}_{F_{1}^{K}}\left[\rho_{F_{1}^{K}}(0) a_{k^{\prime},-\sigma}^{\dagger}(t) a_{k, \sigma}(t) c_{d, \sigma}^{\dagger}(t) c_{d,-\sigma}(t)\right]+\right. \\
\left.\quad+J_{k^{\prime} k}(t) \operatorname{Tr}_{F_{1}^{K}}\left[\rho_{F_{1}^{K}}(0) a_{k^{\prime}, \sigma}^{\dagger}(t) a_{k, \sigma}(t) c_{d, \sigma}^{\dagger}(t) c_{d, \sigma}(t)\right]\right\}
\end{aligned}
$$

where $\rho_{F_{1}^{K}}(0)$ is the density matrix of the system at the zeroth instant of time (compare with Eq. (11)). As usual, the average is taken over the physical subspace $F_{1}^{K}$ of the full Hilbert space for the Kondo model, and the subscript $F_{1}^{K}$ on the right - hand side implies that operators and traces are performed within this subspace. Unlike the physical subspace for the Anderson model (section 1 ) which we referred to as $F_{1}^{A}$, the subspace $F_{1}^{K}$ is defined by the condition that $Q_{K}=1$, where

$$
Q_{K} \equiv \sum_{\sigma} c_{d, \sigma}^{\dagger} c_{d, \sigma}
$$

Definition of the physical subspace for the Anderson model, i.e. the condition $Q_{A}=1$ (see Eq. (10)) fixed the total number of slave particles to be equal to unity, thus, allowing both unoccupied and single - occupied states of the dot. The condition $Q_{K}=1$, on the other hand, enforces single occupation of the dot.

In order to get rid of the constraint to the subspace $F_{1}^{K}$ we adapt the method proposed by Coleman 1 for the analogous problem in the Anderson model, to be used also in the Kondo model. As in section II we combine it with the Schwinger - Keldysh non - equilibrium Green function technique. First, we introduce a grand - canonical Hamiltonian $H_{K}+\lambda Q_{K}$. The limit of infinite $\lambda$ is to be taken at the end of the calculation. Then we go to the interaction representation considering the exchange interaction as a perturbation and the rest of the Hamiltonian, i.e. $\sum_{k \in L, R ; \sigma} \epsilon_{k} a_{k, \sigma}^{\dagger} a_{k, \sigma}+\lambda Q_{K}$ as the unperturbed part. We notice that $Q_{K}$ can be freely added to or subtracted from the Hamiltonian in the definition of the interaction representation since it commutes with all parts of $H_{K}$, although it is important in 
the statistical average as we proceed to show below. Within the interaction representation, equation (47) for the current now reads,

$$
\begin{gathered}
I(t)=-\lim _{t^{\prime} \rightarrow t+0} \frac{e}{\hbar} \sum_{k^{\prime} \in L, k \in R ; \sigma} \operatorname{Im}\left\{J_{k^{\prime} k}(t) \operatorname{Tr}_{F_{1}^{K}}\left[\rho_{F_{1}^{K}}(0) \hat{T}_{p}\left(a_{k^{\prime},-\sigma}^{\dagger}\left(t_{+}\right) a_{k, \sigma}\left(t_{+}^{\prime}\right) c_{d, \sigma}^{\dagger}\left(t_{-}^{\prime}\right) c_{d,-\sigma}\left(t_{-}\right) S_{p}\right)\right]+\right. \\
\left.+J_{k^{\prime} k}(t) \operatorname{Tr}_{F_{1}^{K}}\left[\rho_{F_{1}^{K}}(0) \hat{T}_{p}\left(a_{k^{\prime}, \sigma}^{\dagger}\left(t_{+}\right) a_{k, \sigma}\left(t_{+}^{\prime}\right) c_{d, \sigma}^{\dagger}\left(t_{-}^{\prime}\right) c_{d, \sigma}\left(t_{-}\right) S_{p}\right)\right]\right\}
\end{gathered}
$$

where $S_{p}$ and $\hat{T}_{p}$ are, respectively, the $\mathrm{S}$ - matrix and the time - ordering operator on the closed time - path. In this equation and hereafter, the operators $a_{k, \sigma}^{\dagger}(t), a_{k, \sigma}(t), c_{d, \sigma}^{\dagger}(t)$ and $c_{d, \sigma}(t)$ are defined in the interaction representation. The choice of points $t_{+}, t_{-}, t_{+}^{\prime}$ and $t_{-}^{\prime}$ on the closed time path (see Fig. 5) assures proper ordering of these operators by the operator $\hat{T}_{p}$. As in section \I the initial distribution of the system in the full Hilbert space (before tunneling was switched on) is chosen to be an equilibrium one. It corresponds to a grand - canonical ensemble with the "chemical potential" $-\lambda$. Instead of the operator $O_{A}$ we consider here the operator $O_{K}$ which is defined by the equation,

$$
O_{K} \equiv \hat{T}_{p}\left[\left(a_{k^{\prime},-\sigma}^{\dagger}\left(t_{+}\right) a_{k, \sigma}\left(t_{+}^{\prime}\right) c_{d, \sigma}^{\dagger}\left(t_{-}^{\prime}\right) c_{d,-\sigma}\left(t_{-}\right)+a_{k^{\prime}, \sigma}^{\dagger}\left(t_{+}\right) a_{k, \sigma}\left(t_{+}^{\prime}\right) c_{d, \sigma}^{\dagger}\left(t_{-}^{\prime}\right) c_{d, \sigma}\left(t_{-}\right)\right) S_{p}\right]
$$

Considering expectation values of the operators $O_{K} Q_{K}$ and $Q_{K}$ within the full Hilbert space and repeating the steps leading from Eq. (13) to Eq. (17) (see equations (15) - (16) and explanations therein) we obtain the following expression for the current:

$$
\begin{aligned}
& I(t)=-\frac{e}{\hbar} \sum_{k^{\prime} \in L, k \in R ; \sigma} \operatorname{Im}_{t^{\prime} \rightarrow t+0} \lim _{\lambda \rightarrow \infty}\{ \\
& J_{k^{\prime} k}(t) \frac{\left\langle\hat{T}_{p}\left[a_{k^{\prime},-\sigma}^{\dagger}\left(t_{+}\right) a_{k, \sigma}\left(t_{+}^{\prime}\right) c_{d, \sigma}^{\dagger}\left(t_{-}^{\prime}\right) c_{d,-\sigma}\left(t_{-}\right) S_{p}\right]\right\rangle}{\left\langle Q_{K}\right\rangle}+ \\
&\left.+J_{k^{\prime} k}(t) \frac{\left\langle\hat{T}_{p}\left[a_{k^{\prime}, \sigma}^{\dagger}\left(t_{+}\right) a_{k, \sigma}\left(t_{+}^{\prime}\right) c_{d, \sigma}^{\dagger}\left(t_{-}^{\prime}\right) c_{d, \sigma}\left(t_{-}\right) S_{p}\right]\right\rangle}{\left\langle Q_{K}\right\rangle}\right\},
\end{aligned}
$$

Since the averages in this equation are taken in the full Hilbert space it is amenable for perturbation expansion.

As in section [1] and Ref. 18, we assume that calculation of the tunneling current to lowest order in the tunneling strength which encodes the Kondo effect (i.e. $J_{k^{\prime} k}^{3}$ ) can be done using unperturbed populations of the energy levels. This assumption seems more 
natural here because the number of fermions in the dot is completely fixed by the constraint to the subspace $F_{1}^{K}$. It is not the case in the Anderson model where slave fermions can convert into slave bosons and vise versa within the same subspace $F_{1}^{A}$. Moreover, two levels with different spins have equal occupation numbers after performing an ensemble (thermal) averaging. Therefore, in the Kondo model this assumption concerns only occupation of different energy levels in the reservoirs. The denominator in equation (50) can therefore be easily calculated, that is,

$$
\left\langle Q_{K}\right\rangle=2 n_{d} \stackrel{\lambda \rightarrow \infty}{\longrightarrow} 2 e^{-\beta \lambda},
$$

where $n_{d}=1 /[\exp (\beta \lambda)+1]$ is the Fermi function for the dot electrons in the grand canonical ensemble. In fact, as far as the dot electrons are considered in the grand - canonical ensemble, they are not real particles any more. For example, one might notice that in the physical limit $\lambda \rightarrow \infty$ their Fermi function $n_{d}$ tends to zero. They might be better called "auxiliary fermions" or "slave fermions". To avoid confusion with slave fermions of section [1] we prefer to refer to them hereafter as "dot fermions".

\section{B. Diagrammatic expansion of the current}

In the following we formulate a diagrammatic technique to expand the numerator of Eq. (50) and obtain the current. We skip the detailed derivation and present only its main steps and then present an explicit formulation of the pertinent diagrammatic rules. The strategy is to start from perturbation expansion of Eq. (50) on the closed time - path. Next, transformation of the resulting expression is performed, first to a single - time branch and, then, to the physical representations of the non-equilibrium Green functions. Time - translation invariance of the unperturbed Green functions allows us to use the Fourier transform (for every Green function separately) and write integrals in the energy representation. The rest of this procedure will be explained along the course of formulation below.

Equation (50) expresses the current in terms of a pair of two - particle Green functions. Therefore, in this section we prefer to draw the diagrams in the standard form excepted for 
Green functions in textbooks (see Fig. 6). Alternatively, we could connect all the external lines in one "external vertex" as we did in section II], thus, obtaining diagrams of a circular shape. The difference is, of course, purely superficial. It influences only the rules of drawing, i.e. the first item of the diagrammatic rules formulated below.

Here are the rules for drawing the diagrams and writing down the corresponding analytical expressions:

1. Draw a line corresponding to a propagator of a dot fermion. In Fig. 6 we used a dashed line for its notation. The diagram must include only one such line, since a presence of two or more lines of this type implies $Q_{K} \geq 2$. It would produce contribution to the numerator of equation (50) of second or higher order in powers of $\exp (-\beta \lambda)$. The contribution to the current would then be of first or higher order in powers of $\exp (-\beta \lambda)$, which will vanish at $\lambda \rightarrow \infty$. Attach $m-1$ points to the dot - fermion line, where $m$ is the power of $J_{k^{\prime} k}$ which the contribution of the diagram being considered is supposed to have in the expression for the current. These points represent vertices. Connect them by lead - electron lines leaving two loose ends corresponding to the external operators $a_{k^{\prime}}^{\dagger}$ and $a_{k}$ in Eq. (50). In Fig. 6 we used solid lines to denote propagators of lead electrons. In the present work we have considered diagrams for $m=2$ (Fig. 6 $\mathrm{C}$ ) and $m=3$ (Fig. 6A,B,D).

2. Introduce the following $2 \times 2$ (Keldysh) matrices for every lead - electron and dot fermion line respectively:

$$
g_{k \sigma}(\epsilon) \equiv\left(\begin{array}{cc}
0 & g_{k \sigma}^{a}(\epsilon) \\
g_{k \sigma}^{r}(\epsilon) & g_{k \sigma}^{c}(\epsilon)
\end{array}\right), \xi_{\sigma}(\omega) \equiv\left(\begin{array}{cc}
0 & \xi_{\sigma}^{a}(\omega) \\
\xi_{\sigma}^{r}(\omega) & \xi_{\sigma}^{c}(\omega)
\end{array}\right) .
$$

The indices "r", "a" and "c" denote the retarded, advanced and correlation Green functions which are explicitly defined as follows:

$$
\begin{aligned}
g_{k \sigma}^{r(a)}(\epsilon) & =\frac{1}{\epsilon-\epsilon_{k} \pm i \gamma} \\
g_{k \sigma}^{c}(\epsilon) & =\left[1-2 f\left(\epsilon_{k}\right)\right]\left[g_{k \sigma}^{r}(\epsilon)-g_{k \sigma}^{a}(\epsilon)\right]
\end{aligned}
$$




$$
\begin{aligned}
\xi_{\sigma}^{r(a)}(\omega) & =\frac{1}{\omega-\lambda \pm i \gamma} \\
\xi_{\sigma}^{c}(\omega) & =[1-2 \exp (-\beta \lambda)]\left[\xi_{\sigma}^{r}(\omega)-\xi_{\sigma}^{a}(\omega)\right]
\end{aligned}
$$

where $f\left(\epsilon_{k}\right)=1 /\left[\exp \left(\beta \epsilon_{k}\right)+1\right]$ is Fermi function for lead electrons, the factor $\exp (-\beta \lambda)$ is the limiting case of the dot - fermion Fermi function $n_{d}=1 /[\exp (\beta \lambda)+1]$ at large $\lambda$. As in section $\llbracket$ the matrices (52) emerge as a result of the passage from the closed time - path to the normal time path before the transformation to the energy representation is carried out. Note that the Lagrange multiplier $\lambda$ appears explicitly in the definition of $\xi^{r(a)}$ although consideration of energy conservation shows that, in fact, it could be absorbed into a shift of $\omega$ in all Green functions for the dot - fermions.

3. For every vertex, introduce the following tensor:

$$
\tilde{J}_{k_{\text {out }} k_{i n}} J_{s}\left(\frac{W_{\left(k_{\text {out }} k_{i n}\right)}}{\hbar \Omega}\right)(-1)^{s} \eta_{i j}^{i^{\prime} j^{\prime}}
$$

where $k_{\text {in }}$ and $k_{\text {out }}$ are momenta of incoming and outgoing lead electrons respectively, and $\tilde{J}_{k_{\text {out }} k_{\text {in }}}$ means a matrix element (given by equation (45)). Further, $J_{s}\left(W_{\left(k_{\text {out }} k_{i n}\right)} /(\hbar \Omega)\right)$ are Bessel functions in which the order $s$ is a number of photons absorbed in this vertex (clearly, it can be negative), and

$$
W_{\left(k_{\text {out }} k_{\text {in }}\right)}=\left\{\begin{array}{ll}
W, & \text { if } k_{\text {out }} \in L, k_{\text {in }} \in R \\
-W, & \text { if } k_{\text {out }} \in R, k_{\text {in }} \in L \\
0, & \text { if } k_{\text {out }}, k_{\text {in }} \in L \text { or } k_{\text {out }}, k_{\text {in }} \in R
\end{array} .\right.
$$

It is useful at this point to recall the origin of the appearance of the Bessel functions, starting from the expansion of the time - dependent matrix elements $J_{k_{\text {out }} k_{\text {in }}}(t)$ (see equations (44) and (43)):

$$
\begin{aligned}
& J_{k_{\text {out }} k_{\text {in }}}(t)=\tilde{J}_{k_{\text {out }} k_{\text {in }}} e^{\frac{i}{\hbar}\left[\phi_{\left(k_{\text {out }} k_{\text {in }}\right)} t+i W_{\left(k_{\text {out }} k_{\text {in }}\right)} /(\hbar \Omega) \sin (\Omega t+\alpha)-\beta_{\left(k_{\text {out }} k_{\text {in }}\right)}\right)} \\
& =\tilde{J}_{k_{\text {out }} k_{\text {in }}} \sum_{s=-\infty}^{\infty} J_{s}\left(\frac{W_{\left(k_{\text {out }} k_{\text {in }}\right)}}{\hbar \Omega}\right)(-1)^{s} e^{\frac{i}{\hbar}\left[\phi_{\left(k_{\text {out }} k_{\text {in }}\right)} t-s \Omega t-s \alpha-\beta_{\left(k_{\text {out }} k_{\text {in }}\right)}\right)}
\end{aligned}
$$


The symbols $\left(k_{\text {out }} k_{i n}\right)$ here, as throughout the present manuscript, denote the leads to which $k_{\text {in }}$ and $k_{\text {out }}$ belong, that is, $L R, R L, L L$ or $R R$. The constant phases $\beta_{\left(k_{\text {out }} k_{i n}\right)}$ cancel when equation (56) is substituted into expressions for the diagrams. The tensor $\eta$ is now a tensor of the fourth rank (unlike in section [II) because it connects four Keldysh matrices. It is written explicitly as,

$$
\eta_{i j}^{i^{\prime} j^{\prime}}=\sum_{m^{\prime}, n^{\prime}, m, n} R_{i^{\prime} m^{\prime}}^{-1} R_{j^{\prime} n^{\prime}}^{-1} \sigma_{m^{\prime} n^{\prime}}^{(3)} \delta_{m^{\prime} m} \delta_{m^{\prime} n} R_{m i} R_{n j},
$$

where $R=\frac{1}{\sqrt{2}}\left(\begin{array}{cc}1 & 1 \\ -1 & 1\end{array}\right), R^{-1}=\frac{1}{\sqrt{2}}\left(\begin{array}{cc}1 & -1 \\ 1 & 1\end{array}\right), \sigma_{m^{\prime} n^{\prime}}^{(3)}=\left(\begin{array}{cc}1 & 0 \\ 0 & -1\end{array}\right)$. The third Pauli matrix $\sigma_{m^{\prime} n^{\prime}}^{(3)}$ appears here because of the transition from the closed - time path to the single - time representation (transformation from integration on the backward oriented time branch to integration over normal time axis requires a change of sign). Transformation to the physical representation employs the matrices $R$ and $R^{-1}$.

4. Close around the product of matrices and tensors which has been obtained by the following rows (or columns): $2^{-1 / 2}(-1,1)$ for the $g$ - matrices and $2^{-1 / 2}(1,1)$ for the $\xi$ - matrices (see examples below). The origin of these rows is again the transformation from the single - time representation to the physical representation of the Green functions and vertices. Multiply the result by the factor,

$$
\tilde{J}_{k^{\prime} k} J_{n+s_{1}+\ldots+s_{m-1}}\left(\frac{W_{\left(k^{\prime} k\right)}}{\hbar \Omega}\right)
$$

This factor emerges out of the matrix element $J_{k^{\prime} k}(t)$ in equation (50) by means of the expansion 56 (while $s$ is replaced by $-s$ ). Summation over $n$ is carried out in equation (60) below.

5. Conserve energy in every vertex taking into account absorption of $s$ photons and the energy $\phi^{d c}$ gained (lost) by an electron going from left to right (from right to left).

6. Conservation of spin is somewhat delicate. There are two terms in the Hamiltonian (46) and, correspondingly, two terms in the expression for the current (50). They 
express spin - flip and normal scattering processes. Therefore, conservation of spin in every vertex should take into account two possibilities:

(a) if incoming spins are opposite, then, spin flip occurs,

(b) if incoming spins are equal, then, both outgoing spins are the same.

It is important to stress that the same rules have to be applied to the whole diagram, otherwise it can not contribute to the current (50). Namely,

(a) if incoming spins $\left(\sigma_{e}^{\prime}\right.$ and $\sigma_{f}^{\prime}$ in Fig. 6) are opposite, then, the outgoing spins have to be reversed (i.e. $\sigma_{e}=\sigma_{f}^{\prime}=-\sigma_{e}^{\prime}$ and $\sigma_{f}=\sigma_{e}^{\prime}=-\sigma_{f}^{\prime}$ ),

(b) if incoming spins are equal, then, outgoing spins have to be the same (i.e. $\sigma_{e}=$ $\left.\sigma_{f}=\sigma_{e}^{\prime}=\sigma_{f}^{\prime}\right)$

These rules prohibit the spin - flip diagram of type B in Fig. 6. Indeed, spin - flip in the first vertex can happen only if $\sigma_{e}^{\prime}=-\sigma_{f}^{\prime}$. Then, $\sigma_{e, 1}=-\sigma_{f, 1}=-\sigma_{e}^{\prime}=\sigma_{f}^{\prime}$, and spin - flip should occur in the second vertex as well. Consequently, $\sigma_{e}=-\sigma_{f}=\sigma_{e}^{\prime}=-\sigma_{f}^{\prime}$. This contribution is therefore forbidden. The only diagram of type B which survives is the one with $\sigma_{e}=\sigma_{f}=\sigma_{e, 1}=\sigma_{f, 1}=\sigma_{e}^{\prime}=\sigma_{f}^{\prime}$. On the other hand, four diagrams of type A are allowed. Namely, the following equalities $\sigma_{e, 1}=\sigma_{f, 1}, \sigma_{e}^{\prime}=\sigma_{f}$ and $\sigma_{f}^{\prime}=\sigma_{e}$ should hold. All the combinations which are left are allowed, that is, $\sigma_{e}= \pm \sigma_{f}$ and $\sigma_{f, 1}= \pm \sigma_{f}$

7. Integrate over energy and momentum and sum over spin. Remember that, according to Eq. (50) the current flow starts from the left lead, i.e. $k^{\prime} \in L$, and ends in the right lead, i.e. $k \in R$. The other momenta might belong to any lead: $k_{1}, \ldots, k_{m-2} \in L, R$. Sum over numbers of absorbed photons $s_{1}, \ldots, s_{m-1}$.

8. Multiply the result by the factor

$$
\frac{i e}{\hbar\left\langle Q_{K}\right\rangle}(-i)^{m-1}(i)^{2 m}(-1)^{F}(2 \pi)^{-(m+1)}
$$


where $F$ is the number of closed electron - fermion loops. The first factor in the above expression comes from equation (50), while the second one reminds us of the $m-1$ order of the expansion of the $S$ - matrix. Employing Wick's theorem in the perturbation series requires pairings of creation - annihilation operators which are then expressed in terms of Green functions. This is the origin of the next two factors. The last one emerges following Fourier transforms.

9. Take the limit $\lambda \rightarrow \infty$.

This completes the list of rules for drawing and calculating diagrams in the time - dependent Kondo model.

\section{Explicit expression for the tunneling current}

Let us denote the expression obtained through the above rules for a certain diagram (or after summing of expressions for diagrams up to a certain order) as $\Upsilon_{K}(n)$. The number $n$ has the meaning of the total number of photons absorbed or emitted due to the tunneling process (it was introduced above in the forth item of the diagrammatic rules). The time dependent current is then given by,

$$
\begin{aligned}
I(t) & =\frac{1}{2} I_{0}+\sum_{n=1}^{+\infty}\left|I_{n}\right| \cos \left(n \Omega t+n \alpha+\arg I_{n}\right), \\
I_{n} & \equiv \Upsilon_{K}(n)+\Upsilon_{K}^{*}(-n) .
\end{aligned}
$$

As an example, an expression that is obtained using the above diagrammatic rules for diagram A in Fig. (6) reads,

$$
\begin{aligned}
\Upsilon_{K}^{A}(n)= & -\frac{i e}{\hbar} \sum_{s_{1}, s_{2}} J_{n+s_{1}+s_{2}}\left(\frac{W}{\hbar \Omega}\right) J_{s_{1}}\left(\frac{W_{\left(k_{1}, k^{\prime}\right)}}{\hbar \Omega}\right) J_{s_{2}}\left(\frac{W_{\left(k, k_{1}\right)}}{\hbar \Omega}\right)(-1)^{s_{1}+s_{2}} \\
& \cdot \sum_{k^{\prime} \in L ; k \in R ; k_{1} \in L, R ; \sigma, \sigma^{\prime}, \sigma_{1}} \tilde{J}_{k^{\prime} k} \tilde{J}_{k k_{1}} \tilde{J}_{k_{1} k^{\prime}} \frac{1}{4} \frac{1}{(2 \pi)^{4}} \iiint \int d \epsilon_{1} d \omega d \omega^{\prime} d \omega_{1} \\
& \cdot \lim _{\lambda \rightarrow \infty} \frac{1}{\left\langle Q_{K}\right\rangle} \sum_{i^{\prime}, j^{\prime}, i, j, i_{1}^{\prime}, \ldots, j_{2}}(-1,1)_{j} g_{k \sigma^{\prime}}^{j j_{2}^{\prime}}\left(\epsilon_{1}+\omega^{\prime}-\omega_{1}+\phi_{\left(k_{1} k\right)}+s_{2} \hbar \Omega\right)
\end{aligned}
$$




$$
\begin{gathered}
\cdot \xi_{\sigma_{1}}^{i_{1} i_{2}^{\prime}}\left(\omega_{1}\right) \eta_{i_{2} j_{2}}^{i_{2}^{\prime} j_{2}^{\prime}} g_{k_{1} \sigma_{1}}^{j_{2} j_{1}^{\prime}}\left(\epsilon_{1}\right) \xi_{\sigma^{\prime}}^{i_{2} i^{\prime}}\left(\omega^{\prime}\right)\left(\begin{array}{c}
1 \\
1
\end{array}\right)_{i^{\prime}}(1,1)_{i} \xi_{\sigma}^{i i_{1}^{\prime}}(\omega) \\
\cdot \eta_{i_{1} j_{1}}^{i_{1}^{\prime} j_{1}^{\prime}} g_{k^{\prime} \sigma}^{j_{1} j^{\prime}}\left(\epsilon_{1}+\omega-\omega_{1}+\phi_{\left(k_{1} k^{\prime}\right)}-s_{1} \hbar \Omega\right)\left(\begin{array}{c}
-1 \\
1
\end{array}\right)_{j^{\prime}} .
\end{gathered}
$$

A useful simplification can be worked out in this equation, noticing that one of the vertices connects electron Green functions of the same lead. Indeed, if $k_{1} \in L$, then $k_{1}$ and $k^{\prime}$ belong to the same lead, while if $k_{1} \in R$, then $k_{1}$ and $k$ belong to the same lead. Therefore, according to Eq. (55), one has $W_{\left(k_{\text {out }} k_{\text {in }}\right)}=0$ in this vertex. Using the identity, $J_{s}(0)=\delta_{s, 0}$, we can get rid of one of the Bessel functions. Using also the relation $J_{s}(-x)=(-1)^{s} J_{s}(x)$ we obtain,

$$
\begin{aligned}
& \Upsilon_{K}^{A}(n)=-\frac{i e}{\hbar} \sum_{s} J_{n+s}\left(\frac{W}{\hbar \Omega}\right) J_{s}\left(\frac{W}{\hbar \Omega}\right)_{k^{\prime} \in L ; k \in R ; k_{1} \in L, R ; \sigma, \sigma^{\prime}, \sigma_{1}} \sum_{k^{\prime} k} \tilde{J}_{k k_{1}} \tilde{J}_{k_{1} k^{\prime}} \\
& \text {. } \lim _{\lambda \rightarrow \infty} \frac{1}{\left\langle Q_{K}\right\rangle} \frac{1}{4} \frac{1}{(2 \pi)^{4}} \iiint \int d \epsilon_{1} d \omega d \omega^{\prime} d \omega_{1} \\
& \sum_{i^{\prime}, j^{\prime}, i, j, i_{1}^{\prime}, \ldots, j_{2}}(-1,1)_{j} g_{k \sigma^{\prime}}^{j j_{2}^{\prime}}\left(\epsilon_{1}+\omega^{\prime}-\omega_{1}+\phi_{\left(k_{1} k\right)}+s_{2} \hbar \Omega\right) \\
& \cdot \xi_{\sigma_{1}}^{i_{1} i_{2}^{\prime}}\left(\omega_{1}\right) \eta_{i_{2} j_{2}}^{i_{2}^{\prime} j_{2}^{\prime}} g_{k_{1} \sigma_{1}}^{j_{2} j_{1}^{\prime}}\left(\epsilon_{1}\right) \xi_{\sigma^{\prime}}^{i_{2} i^{\prime}}\left(\omega^{\prime}\right)\left(\begin{array}{c}
1 \\
1
\end{array}\right)_{i^{\prime}}(1,1)_{i} \xi_{\sigma}^{i i_{1}^{\prime}}(\omega) \\
& \cdot \eta_{i_{1} j_{1}}^{i_{1}^{\prime} j_{1}^{\prime}} g_{k^{\prime} \sigma}^{j_{1} j^{\prime}}\left(\epsilon_{1}+\omega-\omega_{1}+\phi_{\left(k_{1} k^{\prime}\right)}-s_{1} \hbar \Omega\right)\left(\begin{array}{c}
-1 \\
1
\end{array}\right)_{j^{\prime}},
\end{aligned}
$$

where $s=s_{1}$, if $k_{1} \in R$, and $s=s_{2}$, if $k_{1} \in L$.

At this stage, the use of a program for analytical calculations (e.g. Mathematica) is indispensable. The following steps are to be done: (1) the tensor - matrix product is to be expanded and expressions for Green functions (53) are to be substituted, (2) due to the presence of the factor $\left\langle Q_{K}\right\rangle$ in the denominator, only terms of the first order in $\exp (-\beta \lambda)$ must be kept in the numerator (the zero - th order cancel while the terms of higher orders are to be omitted), (3) routine integration of the expression obtained through the above steps over internal variables $\epsilon_{1}, \omega, \omega^{\prime}$ and $\omega_{1}$ is to be carried out. The result is the following: 


$$
\begin{gathered}
\Upsilon_{K}^{A}(n)=\frac{i e}{2 \hbar} \sum_{s} J_{n+s}\left(\frac{W}{\hbar \Omega}\right) J_{s}\left(\frac{W}{\hbar \Omega}\right)_{k^{\prime} \in L ; k \in R ; k_{1} \in L, R ; \sigma, \sigma^{\prime}, \sigma_{1}} \sum_{k^{\prime} k} \tilde{J}_{k k_{1}} \tilde{J}_{k_{1} k^{\prime}} . \\
{\left[\frac{f\left(\epsilon_{k}\right) f\left(\epsilon_{k^{\prime}}\right)\left(\epsilon_{k}-\epsilon_{k^{\prime}}-\phi^{d c}-s \Omega-2 i \gamma\right)}{\left(\epsilon_{k}-\epsilon_{k^{\prime}}-\phi^{d c}-s \Omega-i \gamma\right)\left(\epsilon_{k}-\epsilon_{k_{1}}+\phi_{\left(k k_{1}\right)}-s_{2} \Omega-i \gamma\right)\left(\epsilon_{k^{\prime}}-\epsilon_{k_{1}}+\phi_{\left(k^{\prime} k_{1}\right)}-s_{1} \Omega+i \gamma\right)}-\right.} \\
-\frac{f\left(\epsilon_{k}\right) f\left(\epsilon_{k_{1}}\right)}{\left(\epsilon_{k}-\epsilon_{k^{\prime}}-\phi^{d c}-s \Omega-i \gamma\right)\left(\epsilon_{k^{\prime}}-\epsilon_{k_{1}}+\phi_{\left(k^{\prime} k_{1}\right)}-s_{1} \Omega+i \gamma\right)}+ \\
\left.+\frac{f\left(\epsilon_{k^{\prime}}\right) f\left(\epsilon_{k_{1}}\right)}{\left(\epsilon_{k}-\epsilon_{k^{\prime}}-\phi^{d c}-s \Omega-i \gamma\right)\left(\epsilon_{k}-\epsilon_{k_{1}}+\phi_{\left(k k_{1}\right)}-s_{2} \Omega-i \gamma\right)}\right]
\end{gathered}
$$

We assume now that $J_{k^{\prime} k}$ depend only on the leads to which $k^{\prime}$ and $k$ belong, independently of the values of $k^{\prime}$ and $k$ (hence there are just three coupling - strength constants: $J_{L L}, J_{R R}$ and $J_{L R}$, where $\left.J_{L(R) R(L)}=V_{L(R)} V_{R(L)}^{*} /\left|\epsilon_{d, \sigma}\right|\right)$. Furthermore, we assume that the densities of states in the leads $\left(\rho\left(\epsilon_{k}\right), k \in L, R\right)$, are roughly constant on the energy scale of $\phi^{d c}, \Omega$ and $W$ (rigorously speaking, only the combinations $\rho\left(\epsilon_{k^{\prime}}\right)^{1 / 2} J_{k^{\prime} k}(t) \rho\left(\epsilon_{k}\right)^{1 / 2}$, where $k, k^{\prime} \in L, R$, need to be constant). Then the above expression greatly simplifies since integration over one of $k, k^{\prime}$ or $k_{1}$ can be carried out. Using also a simple relation,

$$
\tilde{J}_{k^{\prime} k} \tilde{J}_{k k_{1}} \tilde{J}_{k_{1} k^{\prime}}=\left|\tilde{J}_{L R}\right|^{2} \tilde{J}_{\left(k_{1} k_{1}\right)}
$$

we get an expression,

$$
\begin{aligned}
\Upsilon_{K}^{A}(n) & =\frac{e}{\hbar} 8 \pi\left|\tilde{J}_{L R}\right|^{2} \rho_{L} \rho_{R}\left(\tilde{J}_{L L} \rho_{L}+\tilde{J}_{R R} \rho_{R}\right) \\
& \cdot \sum_{s} J_{n+s}\left(\frac{W}{\hbar \Omega}\right) J_{s}\left(\frac{W}{\hbar \Omega}\right) \iint d \epsilon_{k} d \epsilon_{k^{\prime}} \frac{f\left(\epsilon_{k}\right) f\left(\epsilon_{k^{\prime}}\right)}{\epsilon_{k}-\epsilon_{k^{\prime}}-\phi^{d c}-s \Omega-i \gamma}
\end{aligned}
$$

The factor 8 in this equation arises from summation over spins.

For the diagram B in Fig. 6 the above diagrammatic rules, using $J_{s}(-x)=(-1)^{s} J_{s}(x)$ and $J_{s}(0)=\delta_{s, 0}$, yield the following expression:

$$
\begin{aligned}
\Upsilon_{K}^{B}(n)= & \frac{i e}{\hbar} \sum_{s} J_{n+s}\left(\frac{W}{\hbar \Omega}\right) J_{s}\left(\frac{W}{\hbar \Omega}\right) \sum_{k^{\prime} \in L ; k \in R ; k_{1} \in L, R ; \sigma} \tilde{J}_{k^{\prime} k} \tilde{J}_{k k_{1}} \tilde{J}_{k_{1} k^{\prime}} \\
& \cdot \lim _{\lambda \rightarrow \infty} \frac{1}{\left\langle Q_{K}\right\rangle} \frac{1}{4} \frac{1}{(2 \pi)^{4}} \iiint \int d \epsilon_{1} d \omega d \omega^{\prime} d \omega_{1} \\
& \cdot \sum_{i^{\prime}, j^{\prime}, i, j, i_{1}^{\prime}, \ldots, j_{2}}(-1,1)_{j} g_{k \sigma}^{j j_{2}^{\prime}}\left(\epsilon_{1}-\omega+\omega_{1}+\phi_{\left(k_{1} k\right)}+s_{2} \hbar \Omega\right)
\end{aligned}
$$




$$
\begin{aligned}
& \cdot(1,1)_{i} \xi_{\sigma}^{i i_{2}^{\prime}}(\omega) \eta_{i_{2} j_{2}}^{i_{2}^{\prime} j_{2}^{\prime}} g_{k_{1} \sigma}^{j_{2 j} j_{1}^{\prime}}\left(\epsilon_{1}\right) \xi_{\sigma}^{i_{2} i_{1}^{\prime}}\left(\omega_{1}\right) \eta_{i_{1} j_{1}}^{i_{1}^{\prime} j_{1}^{\prime}} \\
& \cdot g_{k^{\prime} \sigma}^{j_{j} j^{\prime}}\left(\epsilon_{1}-\omega^{\prime}+\omega_{1}+\phi_{\left(k_{1} k^{\prime}\right)}-s_{1} \hbar \Omega\right)\left(\begin{array}{c}
-1 \\
1
\end{array}\right)_{j^{\prime}} \xi_{\sigma}^{i_{1} i^{\prime}}\left(\omega^{\prime}\right)\left(\begin{array}{l}
1 \\
1
\end{array}\right)_{i^{\prime}} .
\end{aligned}
$$

Repeating the steps which led from Eq. (62) to Eq. (65) we obtain,

$$
\begin{aligned}
\Upsilon_{K}^{B}(n) & =\frac{e}{\hbar} 2 \pi\left|\tilde{J}_{L R}\right|^{2} \rho_{L} \rho_{R}\left(\tilde{J}_{L L} \rho_{L}+\tilde{J}_{R R} \rho_{R}\right) \\
& \cdot \sum_{s} J_{n+s}\left(\frac{W}{\hbar \Omega}\right) J_{s}\left(\frac{W}{\hbar \Omega}\right) \iint d \epsilon_{k} d \epsilon_{k^{\prime}} \frac{f\left(\epsilon_{k}\right) f\left(\epsilon_{k^{\prime}}\right)}{\epsilon_{k}-\epsilon_{k^{\prime}}-\phi^{d c}-s \Omega-i \gamma} .
\end{aligned}
$$

We omitted in this expression some terms which cancel out when substituted into expression for the current (60). Note that spin - conservation rules discussed above allow only one combination of spins for this diagram, namely, all spin projections are identical. It leads to a factor 2 in the above expression instead of 8 in Eq. (65).

For the diagram $\mathrm{C}$ in Fig. 6 we obtain, using the above diagrammatic rules and $J_{s}(-x)=$ $(-1)^{s} J_{s}(x)$ an expression of second order in $\tilde{J}_{k^{\prime} k} \rho$,

$$
\begin{aligned}
\Upsilon_{K}^{C}(n)= & \frac{i e}{\hbar} \sum_{s} J_{n+s}\left(\frac{W}{\hbar \Omega}\right) J_{s}\left(\frac{W}{\hbar \Omega}\right)_{k^{\prime} \in L ; k \in R ; \sigma, \sigma_{1}}\left|\tilde{J}_{k^{\prime} k}\right|^{2} \\
& \cdot \lim _{\lambda \rightarrow \infty} \frac{1}{\left\langle Q_{K}\right\rangle} \frac{1}{4} \frac{1}{(2 \pi)^{3}} \iiint \int d \epsilon^{\prime} d \omega d \omega^{\prime} \\
& \cdot \sum_{i^{\prime}, j^{\prime}, i, j, i_{1}^{\prime}, \ldots, j_{1}}(-1,1)_{j} g_{k \sigma}^{j j_{1}^{\prime}}\left(\epsilon^{\prime}-\omega+\omega^{\prime}+\phi^{d c}+s \hbar \Omega\right) \\
& \cdot(1,1)_{i} \xi_{\sigma_{1}}^{i i_{1}^{\prime}}(\omega) \eta_{i_{1} j_{1}}^{i_{1}^{\prime} j_{1}^{\prime}} g_{k^{\prime} \sigma_{1}}^{j_{1} j^{\prime}}\left(\epsilon^{\prime}\right)\left(\begin{array}{c}
-1 \\
1
\end{array}\right)_{j^{\prime}} \xi_{\sigma}^{i_{1} i^{\prime}}\left(\omega^{\prime}\right)\left(\begin{array}{l}
1 \\
1
\end{array}\right)_{i^{\prime}} .
\end{aligned}
$$

Making the same steps as above we obtain a very simple equation:

$$
\begin{aligned}
\Upsilon_{K}^{C}(n) & =\frac{e}{\hbar} 2 \pi\left|\tilde{J}_{L R}\right|^{2} \rho_{L} \rho_{R} \\
& \cdot \sum_{s} J_{n+s}\left(\frac{W}{\hbar \Omega}\right) J_{s}\left(\frac{W}{\hbar \Omega}\right) \cdot\left(\phi^{d c}+s \hbar \Omega+i \text { const }\right) .
\end{aligned}
$$

The last term (i.e. $i \cdot$ const) cancels when substituted into expression for the current $(60)$.

In order to calculate contribution of diagram $\mathrm{D}$ it is better to stay in the closed time - path representation. Then one can easily notice that it can be expressed through the contribution of diagram $\mathrm{C}$ in the following manner: 


$$
\Upsilon_{K}^{D}(n)=\Upsilon_{K}^{C}(n) \int_{p} d t_{p} \sum_{k_{1} \in L, R} J_{k_{1} k_{1}}(t) n_{k_{1}}
$$

where $n_{k_{1}} \equiv\left\langle a_{k_{1}}^{\dagger} a_{k_{1}}\right\rangle=f\left(\epsilon_{k}\right)$. The matrix elements $J_{k_{1} k_{1}}(t)$ do not depend on the branch to which $t$ belongs, i.e. whether $t_{p}=t_{-}$or $t_{p}=t_{-}$, while $n_{k_{1}}$ does not depend on time at all. So the integrals over the normal and the backward oriented time branches cancel. The contribution of this diagram to the current simply vanishes.

Further progress employs the identities

$$
\begin{aligned}
\sum_{s} J_{s}(x) J_{s+n}(x) & =\delta_{n, 0} \\
\sum_{s} s J_{s}(x) J_{s+n}(x) & =\frac{x}{2}\left(\delta_{n, 1}+\delta_{n,-1}\right) .
\end{aligned}
$$

Substituting Eq. (69) into Eq. (60) and using these equations we obtain for the contribution of diagram $\mathrm{C}$ to the current $\left(I^{(2)}(t)\right)$ the following simple expression:

$$
I^{(2)}(t)=C_{2}\left[\phi^{d c}+W \cos (\Omega t+\alpha)\right]
$$

where $C_{2} \equiv \frac{e}{\hbar} \pi\left|\tilde{J}_{L R}\right|^{2} \rho_{L} \rho_{R}$. This is the only contribution which is of second order in $J_{k^{\prime} k}(t) \rho$. There appear only $D C$ and the first harmonic. The higher harmonics are not generated in this order.

Finally we sum contributions of all the diagrams and get the following equations for the tunneling current:

$$
\begin{aligned}
I(t) & =I^{(2)}(t)+I^{(3)}(t) \\
I^{(2)}(t) & =C_{2}\left[\phi^{d c}+W \cos (\Omega t+\alpha)\right] \\
I^{(3)}(t) & =\frac{1}{2} I_{0}+\sum_{n=1}^{\infty}\left|I_{n}\right| \cos \left(n \Omega t+n \alpha+\arg I_{n}\right), \\
I_{n} & \equiv C_{3} \sum_{s=-\infty}^{+\infty} J_{s}\left(\frac{W}{\hbar \Omega}\right)\left[J_{s+n}\left(\frac{W}{\hbar \Omega}\right) F\left(\phi^{d c}+s \Omega, T, D\right)+\right. \\
F(\phi, T, D) & =\int_{-D}^{+\infty} \int_{-D}^{+\infty} d \epsilon_{k} d \epsilon_{k^{\prime}} \frac{f\left(\epsilon_{k}\right) f\left(\epsilon_{k^{\prime}}\right)}{\epsilon_{k}-\epsilon_{k^{\prime}}-\phi-i \gamma},
\end{aligned}
$$

where $C_{2}=\frac{e}{\hbar} \pi\left|\tilde{J}_{L R}\right|^{2} \rho_{L} \rho_{R}$ and $C_{3} \equiv \frac{e}{\hbar} 10 \pi\left|\tilde{J}_{L R}\right|^{2} \rho_{L} \rho_{R}\left(\tilde{J}_{L L} \rho_{L}+\tilde{J}_{R R} \rho_{R}\right)$, while $\rho_{L(R)}$ are densities of states in the leads. The quantities $I^{(2)}$ and $I^{(3)}$ express contributions of second 
(diagram C) and third (diagrams A and B) orders in $J_{k^{\prime} k} \rho$ respectively. The cutoff $D$ is equal to the energy difference between the chemical potential and the bottom of the conduction band, while $\gamma$ is an infinitesimally small number. Equations (73) constitute the central formal result of the present section. Equation (73t) is rather convenient for further elaboration (which is our next step) but it can be misleading if a proper care is not taken. Indeed, the cutoffs for the two energy integrations are not independent, and their relation is to be defined carefully. Consider another expression for $F$ where one of the integrals does not require a cutoff:

$$
F(\phi, T, D)=-\frac{1}{2} \operatorname{Re} \iint_{-D}^{+\infty} d \omega d \epsilon \frac{\left[f_{L}(\omega)-f_{R}(\omega)\right]\left[f_{L}(\epsilon)+f_{R}(\epsilon)\right]}{\omega-\epsilon+i \gamma}-i \pi \frac{\phi}{2} \operatorname{coth} \frac{\beta \phi}{2},
$$

Here $f_{L}(\epsilon) \equiv 1 /(\exp [(\epsilon-\phi) / k T]+1)$ and $f_{R}(\epsilon) \equiv 1 /(\exp [\epsilon / k T]+1)$ have the meaning of Fermi functions in the leads (the left lead being shifted by $\phi$ ). Some pure imaginary terms, linear in $\phi$, that do not contribute to the current have been omitted in this expression. The above integral can be written in the same form as in the $D C$ result of Sivan and Wingreen 1 . The physical content of equation (73d) for $I_{n}$ is rather transparent: An alternating field applied to a two - barrier system actually results in a splitting of the leads energy levelsit. Therefore the time - dependent current is a result of interference between " $D C$ - like" contributions, each one of them having an effective bias $\phi^{d c}+s \Omega$ weighted by the appropriate product of Bessel functions 62 . An approximate evaluation of the double integral in the above equations is possible both for the linear $(\phi \ll T)$ and for the nonlinear $(\phi \gg T)$ regimes. It yields,

$$
\operatorname{Re}[F(\phi, T, D)]=\left\{\begin{array}{c}
\phi\left[\ln \frac{D}{k T}+0.26+O(\phi / k T)+O(k T / D)\right], \text { if } \phi \ll k T, \\
\phi\left[\ln \frac{D}{|\phi|}+1+O\left((k T / \phi)^{2}\right)+O(\phi / D)\right], \text { if } \phi \gg k T .
\end{array}\right.
$$

As is well known (see, for instance, Ref. 四), the linear - response perturbation expansion for the Kondo problem breaks down at temperatures below a certain value, defined as the Kondo temperature $T_{K}$. In our calculation it shows as a logarithmic divergence of the function $F$ and, consequently, the amplitude of the current (73d) at $T \rightarrow 0$ in the linear response. It is remarkable, however, that in the nonlinear case on which we focus our attention here 
the function $F$ does not diverge with decreasing temperature. Hence, our results for the nonlinear response are valid even below the Kondo temperature. This is due to the fact that here the non - linear bias plays the role of temperature as the largest low - energy scale.

When the estimate (75) is employed in equations (73) it yields extremely simple expressions for the current. First, it turns out to be very useful for the static non-equilibrium case $(W=0)$, for which we find,

$$
I \approx \phi^{d c} \cdot\left[\begin{array}{c}
C_{2}+0.26 C_{3}+C_{3} \ln \frac{D}{k T}, \text { if } \phi^{d c} \ll T, \\
C_{2}+C_{3}+C_{3} \ln \frac{D}{\left|\phi^{d c}\right|}, \text { if } \phi^{d c} \gg T .
\end{array}\right.
$$

To the best of our knowledge, such a simple expression for a non - equilibrium tunneling current through a Kondo system has not been derived before. We notice that it contains the familiar pattern of the zero - bias anomaly (i.e. a peak in the differential conductance at zero bias).

Next, considering expression (75) we notice that the large factor $\ln D$ appears only in a term which is linear in $\phi$. Using equations $(73 \mathrm{~d})$ and $(71)$, we then find that this factor emerges only in the expressions for the $D C$ and the first harmonic of the $A C$. It means that, in fact, only the direct and the first harmonic are enhanced by the Kondo effect. As for the higher harmonics, the interference of the contributions to the current in equations (73) with different effective biases is destructive. This conclusion is further confirmed by numerical calculation of the whole spectrum of the tunneling current performed using equations (73). In Fig. 7 the spectra (amplitude of the harmonics $I_{n}$ via their number $n$, the value of $1 / 2 I_{0}$ is displayed for the $D C$ ) are shown for both the Kondo and the non - interacting systems. Values of the parameters used for the calculation are listed in the caption. For the non - interacting system we used the equations of Ref. 63 reduced to the case of only one resonant level present in the system. Comparison of the top and bottom parts of the figure clearly shows a significant enhancement of $D C$ and the first harmonic relative to the others in the Kondo system. The ratio of the $D C$ and the first harmonic to the higher harmonics might increase even further if $D$ becomes larger. However, the choice of parameters for Fig. 7 corresponds to a real quantum dot situation 1]. We emphasize that the non - linear 
response is different from the linear one. Namely, (i) the second and the higher harmonics exist although they are small, (ii) the amplitudes of the $D C$ and the first harmonic are not determined any more by the ratio $D / T$ as in the linear response, but rather by the ratio of $D$ to the largest relevant low - energy scale as we show in the following (see equations (77), (81) and (82) below).

Substituting equation (75) into Eq. (73), it is possible, in a few limiting cases, to find very simple expressions for the $D C$ and the first harmonic of the time - dependent tunneling current. Experimentally, the nonlinear Kondo effect is usually revealed as the zero - bias anomaly. In a strong alternating field where many - photon absorption is possible (i.e. $W \gg \Omega, T)$, we get for the differential conductance $\left(\partial I^{d c} / \partial \phi^{d c}\right)$ an expression,

$$
\begin{aligned}
& \frac{\partial I^{d c}}{\partial \phi^{d c}} \approx C_{2}+C_{3}[\ln (D / W)+1]+C_{3} \frac{\Omega}{2 W} \sum_{|q|<W / \Omega} P\left(\phi^{d c} / \Omega-q, T / \Omega\right), \text { if }\left|\phi^{d c}\right| \ll W, \\
& \frac{\partial I^{d c}}{\partial \phi^{d c}} \approx C_{2}+C_{3} \ln \left(D /\left|\phi^{d c}\right|\right), \text { if }\left|\phi^{d c}\right| \gg W,
\end{aligned}
$$

where $P\left(\phi^{d c} / \Omega-q, T / \Omega\right)$ is the shape function for the side peaks at $\phi^{d c}=q \Omega, q$ is integer. We find that,

$P\left(\phi^{d c} / \Omega-q, T / \Omega\right) \approx \frac{1}{2} \ln \frac{\Omega^{2}}{\left(\phi^{d c}-q \Omega\right)^{2}+T^{2}}$, if $\left|\phi^{d c} / \Omega-q\right| \ll 1$ and $|T / \Omega| \ll 1$, while $P\left(\phi^{d c} / \Omega-q, T / \Omega\right)=0$, if $\left|\phi^{d c} / \Omega-q\right|>1$ or $|T / \Omega|>1$.

In a weak alternating field $(W \ll \Omega)$, we obtain,

$$
\frac{\partial I^{d c}}{\partial \phi^{d c}} \approx C_{2}+\frac{C_{3}}{2} \ln \frac{D^{2}}{\left(\phi^{d c}\right)^{2}+T^{2}}+C_{3} \sum_{q \neq 0}\left(\frac{W}{2 \Omega}\right)^{2|q|} \frac{1}{(|q| !)^{2}} P\left(\phi^{d c}-q \Omega, T, D\right),
$$

where the side - peak shape function $P$ is given by,

$$
P\left(\phi^{d c}-q \Omega, T, D\right) \approx \frac{1}{2} \ln \frac{D^{2}}{\left(\phi^{d c}-q \Omega\right)^{2}+T^{2}}
$$

Comparing equation (77) with the static expression $(76)$, we notice that the main peak of the differential conductance (the one at $\phi^{d c}=0$ ) is suppressed in a strong alternating field by the factor approximately equal to $[\ln (D / W)+1] /[\ln (D / T)+0.26]$. The side peaks in 
a strong field are not simple replicas of the central one, as is the case in a weak field. As far as the temperature dependence of the zero bias anomaly for the time - independent response is concerned, we note that the temperature dependence of the side peaks in a weak alternating field is governed by the factor $\ln (D / T)$. In a strong alternating field, however, it is determined by the factor $\ln (\Omega / T)$. Moreover, the amplitudes of the side peaks in a strong alternating field does not decay exponentially with $q$. In fact, it is roughly constant (as long as $q \Omega<W$ ). The half - width of the side peaks in a strong field is approximately equal to $(\Omega T)^{1 / 2}$ instead of $(D T)^{1 / 2}$. The latter feature is quite favorable for experimental observation: since the peaks are rather narrow it is not necessary to go to high frequencies in order to resolve them. On the other hand, their magnitude decreases together with the ratio $\Omega / T$. In Fig. 8 and Fig. 9 the differential $D C$ - conductance (calculated numerically using equations $(73)$ ) is shown versus the bias at different magnitudes of the alternating field. Suppression of the central peak in the zero bias anomaly is readily manifested in both figures. Side peaks do not appear in Fig. 8 where the ratio of the frequency to the temperature is not large enough $(\Omega / T=5)$. They are well pronounced, however, at a tenfold lower temperature (Fig. 9).

Although measurement of an $A C$ with frequencies and amplitudes in the relevant range is not an easy task, it might reveal new interesting features of the Kondo effect. Kondo contribution to the direct tunneling current is usually revealed in an experiment through a special dependence on the parameters (such as $\ln T$ increase of the conductance or the zero - bias anomaly). It was shown above that, as far as the spectrum of the tunneling current is concerned, only the $D C$ and the first harmonic are enhanced by the Kondo effect. This implies that such kind of parameter dependence can be found in the first harmonic as well as in the $D C$ but not in higher harmonics. Employing the approximate expression (75) in Eq. (73) we obtain for the first harmonic of the time - dependent tunneling current $\left(I^{a c}\right)$ in a strong alternating field $(W \gg \Omega)$,

$$
I^{a c} \approx C_{2} W+\frac{2}{3} C_{3} W[\ln (D / W)+2], \text { for } W \gg\left|\phi^{d c}\right|, T
$$




$$
\begin{aligned}
I^{a c} & \approx C_{2} W+\frac{2}{3} C_{3} W\left[\ln \left(D /\left|\phi^{d c}\right|\right)+1 / 3\right], \text { for } W, T \ll\left|\phi^{d c}\right|, \\
I^{a c} & \approx C_{2} W+C_{3} W[\ln (D / T)+1 / 4], \text { for } W,\left|\phi^{d c}\right| \ll T .
\end{aligned}
$$

These expressions appear to be very similar to equations (77) for the $D C$ when $\left|\phi^{d c}\right|$ and $W$ are exchanged. Moreover, differentiating $I^{a c}$ with respect to $W$, we find a peak in the differential $A C$ - conductance at zero $A C$ - bias. The shape of the peak is logarithmic at large $W$, while its height is determined by $\max \left(\left|\phi^{d c}\right|, T\right)$. We infer that this feature of the $A C$ is analogous to the familiar zero bias anomaly in the $D C$. In the same way that the latter is suppressed by the alternating - bias, this "zero alternating - bias anomaly" is suppressed by the direct - bias. In Fig. 10 the differential alternating - conductance calculated using equations (73) is plotted versus alternating - bias $W$ at different values of the direct - bias $\phi^{d c}$. Notice the clear similarity with the zero bias anomaly shown in Fig. 8 and its suppression with alternating - bias. We do not find any side - peaks in the differential $A C$ - conductance. Their traces can be exposed, however, in the dependence of the $A C$ on the direct - bias at low temperatures. In Fig. 11 its derivative $\left(\frac{d I^{a c}}{d \phi^{d c}}\right)$ is drawn versus $\phi^{d c}$ for two temperatures. At low temperature there appear well - pronounced dips (which are actually peaks in its absolute value) at integer multiples of the frequency.

In a weak alternating field $(W \ll \Omega)$ we find,

$$
\begin{aligned}
I^{a c} & \approx C_{2} W+C_{3} W \ln \left(D /\left|\phi^{d c}\right|\right), \text { for } T, \Omega \ll\left|\phi^{d c}\right|, \\
I^{a c} & \approx C_{2} W+C_{3} W[\ln (D / \Omega)+1], \text { for } T,\left|\phi^{d c}\right| \ll \Omega, \\
I^{a c} & \approx C_{2} W+C_{3} W[\ln (D / T)+1 / 4], \text { if }\left|\phi^{d c}\right|, \Omega \ll T .
\end{aligned}
$$

Inspecting equations (76), (77), (81) and (82) we notice that the values of both direct and alternating differential - conductances are basically determined by the logarithm of the ratio of $D$ to the largest relevant low - energy scale. 


\section{CONCLUSIONS}

In the present work the problem of non-equilibrium time - dependent electron tunneling through an interacting system was studied at some depth. The main attention was focused on calculation of time - dependent current in the Kondo regime beyond linear response. A tunneling system in this context is naturally described by the time - dependent Anderson model. Perturbation expansion of the current within this model, specially adapted for systems out of equilibrium was elaborated upon in section [1]. This formalism combines the non-equilibrium Green functions method with a specific approach suggested by Coleman 41 to account for averaging in restricted subspaces which is often encountered in problems involving strongly correlated electron systems. We have accomplished the formal part of perturbation expansion within this model for the time - dependent case. The task of performing detailed calculations turns out to be too formidable. Yet, with a slightly elevated capability of present day workstations and analytic software programs it should be feasible.

We then suggested a way to overcome this problem in section III where we developed a time - dependent version of the Schrieffer - Wolff transformation mapping the time - dependent Anderson model onto a Kondo - type model. The latter is much easier for treatment within perturbation theory. We maintain that it cannot be introduced phenomenologically since proper correlation of the time - dependence between the leads and the Kondo coupling constant $J_{k k^{\prime}}(t)$ has to be taken into account. Non-Equilibrium perturbation technique for calculation of the tunneling current within the time - dependent Kondo model was worked out in section IV. Actual calculations were performed up to the third order in $J_{k k^{\prime}}$ (which corresponds to sixth order in tunneling matrix elements for the Anderson model) yielding extremely simple analytical expressions for the whole spectrum of the tunneling current (see equations (73), (76), (77), (81) and (82)). The nonlinear time - dependent current was found to be an interference sum of " $D C$ - like" contributions, each one with an effective bias altered by the number of absorbed or emitted photons. We stress that our results are valid for the non - linear response both below and above the Kondo temperature although for the linear 
response they are valid only above it.

There are three novel results in the present research. First, it was found that the Kondo effect strongly affects the first harmonic of the alternating tunneling current, no less that it affects the $D C$, while the other harmonics remain relatively small. This result is shown to originate from the interference of $D C$ - like contributions to the current (equation $(73)$ that turn out to be rather destructive for all the harmonics except the $D C$ and the first one. The higher harmonics are of course generated but their amplitudes are relatively small. This result was demonstrated to be remarkably different from that for a non - interacting one level system where all the harmonics emerge together.

Second, it was found that the zero - bias anomaly in the $D C$ is suppressed by an alternating field and displays side-peaks at multiples of the basic frequency. This result can be easily tested experimentally since it is concerned with measurement of the $D C$ in the Kondo regime, which has now been well - established in quantum - dots experiments. Expressions (77) together with Figs. 8 and 9 provide an estimate for the preferred range of parameters of the system.

Third, we found a "zero $A C$ - bias anomaly" in the alternating current, i.e. a peak of the differential $A C$ - conductance at zero $A C$ - bias. This phenomenon is an $A C$ - analog of the familiar zero - bias anomaly of the $D C$. As in the latter one, it is suppressed by the $A C$ - bias, while the former one is suppressed by the $D C$ - bias. There are no side - peaks of the differential $A C$ - conductance, however they have a well - pronounced counterpart in the derivative of the $A C$ with respect to the $D C$ - bias (in the form of dips). We think that this phenomenon shows a pattern of the Kondo effect in the $A C$ yielding a challenging object for an experimental search. Equations (81), (82) and Figs. 10, 11 provide an estimate for the necessary range of parameters. Our results on the spectrum of the current indicate that effects like this one cannot appear at higher harmonics of the time - dependent tunneling current (due to the Kondo effect). We emphasize that expressions (77), (81) and (82), especially the numerical coefficients, are very approximate. They are intended to display the basic dependence of the current on the parameters of the system. Quantitative comparison 
with experiment may be done using the full set of equations $(73)$.

As far as relation to previous relevant works is concerned, we first notice that our analytical results for the $D C$ are consistent with the numerical calculations of Ref. 17. However, being able to consider stronger $A C$ - fields (larger ratio $W / \Omega$ ), we find also an overall suppression of the zero - bias anomaly, beside the appearance of side peaks. As for the Fourier spectrum of the time - dependent tunneling current, we are unable to validate the assumption suggested in Ref. 17 that all the harmonics beside the $D C$ one can be neglected. Rather, the first harmonic is also enhanced, while the second and higher harmonics are generated but they are indeed much smaller than the $D C$ and the first ond 64 . Within a specific model, some author $\$ 21$ obtained current spectrum similar to that of a non - interacting system. We attribute the difference between this result and ours to a quite peculiar choice of parameters used therein.

We believe that further research on time - dependent aspects of the Kondo effect, in particular in quantum dots, is interesting and very timely. Let us mention a few possible directions of future research. First, the methods developed in the present work can be adapted for solution of the problem of nonlinear response of a Kondo system to a combination of alternating magnetic and electric fields. Evolution of the zero - bias anomaly in a magnetic field, contrary to its temperature dependence, seems to be the clearest experimentallyt. $\mathrm{B}$ resolved feature of the non-equilibrium Kondo effect. Calculation of complementary effects in the time - dependent response and carrying out pertinent experiments seem also to be timely. If the effect of a magnetic field can be solely expressed by the Zeeman splitting of the energy level in the dot, that is, $\epsilon_{\sigma= \pm 1}=\epsilon_{0} \pm \Delta \epsilon / 2$, the technique developed in the present work can be easily modified to incorporate it.

It might also be interesting to consider a non - magnetic (also called "orbital") Kondo system 55,66 subject to a strong alternating field. It is believed 67.68 to be realized in some recent experiments 79 in the form of a two - level atomic tunneling system. In this model the conducting electrons interact with an impurity atom which can tunnel between two states. Tunneling of the atom is assisted by the interaction. At sufficiently low temperatures, the 
parameters of the model renormalize so that it becomes equivalent to the 2 - channel Kondo model 69 . Besides the time - dependent shift of the leads which is present also in the 1 channel model and studied in our work, in a two - level atomic system a time - dependent field can cause a change of the effective energy separation between the levels. One possible effect of such a change is a crossover between Fermi - liquid and non - Fermi - liquid behavior (see, for instance, Ref. 68). It is especially appropriate to point out here that application of an $A C$ - field is rather controllable. An appropriate calculation, if followed by an experiment, could then further test the hypothesis that the physics of a 2 - channel Kondo model has been realized in some experiments, 明, a point which has been questioned by some authors 7 .

Consideration of a multilevel Kondo system in an external alternating field looks very attractive. First, it is an appropriate object for quantum dots experiments. Moreover, it was argued 1,28 that the Kondo temperature in such a system can be enhanced by orders of magnitude. This fact could allow an experimental investigation of the strongly correlated regime of the Kondo system. On the other hand, application of a time - dependent field to a multilevel system leads to highly nonlinear tunneling processes, e.g. resonant frequency multiplication 63 . Examination of non-equilibrium transport through such a system in the Kondo regime might reveal new and interesting effects.

In closing, we believe that the physics of strongly correlated particles in strong time dependent external fields and restricted geometries looks to be an important and exciting subject for further research.

Acknowledgments: This research is supported in part by a grant from the Israel Science Foundation under programs Centers of Excellence, and Non-Linear Tunneling, by an American - Israel BSF grant Dynamical Instabilities and by a DIP program Quantum Electronics in Low Dimensional Systems. We would like to thank N.S. Wingreen, A. Golub, Y. Meir, D. Goldhaber - Gordon, L.P. Kouwenhoven, P. Coleman, L.I. Glazman, A. Schiller, K.A. Kikoin, G. Schön and E. Kogan for helpful discussions and comments. 


\section{REFERENCES}

* Electronic address: yshai@bgumail.bgu.ac.il

${ }^{1}$ D. Goldhaber - Gordon et al., Nature, 391, 156 (1998); cond-mat/9807233.

${ }^{2}$ S.M. Cronenwett et al., Science, 281, 540 (1998).

${ }^{3}$ F. Simmel, R.H. Blick, J.P. Kotthaus, W. Wegscheider and M. Bichler, cond-mat/9812153.

${ }^{4}$ A. C. Hewson, The Kondo Problem to Heavy Fermions (Cambridge University Press, Cambridge, 1993).

${ }^{5}$ P. Fulde, Electron Correlations in Molecules and Solids, Ch. 12 (Springer, Berlin, 1991).

${ }^{6}$ S. Gregory, Phys. Rev. Lett., 68, 2070 (1992).

${ }^{7}$ D.C. Ralph, R.A. Buhrman, Phys. Rev. Lett., 69, 2118 (1992).

${ }^{8}$ D.C. Ralph, R.A. Buhrman, Phys. Rev. Lett., 72, 3401 (1994).

${ }^{9}$ R.J.P. Keijsers, O.I. Shklyarevskii and H. van Kempen, Phys. Rev. B, 51, 5628 (1995); Phys. Rev. Lett., 77, 3411 (1996).

${ }^{10}$ I.K.Yanson, V.V. Fisun, R. Hesper, A.V. Khotkevich, J.M. Krans, J.A. Mydosh and J.M. van Ruitenbeek, Phys. Rev. Lett., 74, 302 (1995).

${ }^{11}$ S. Hershfield, J.H. Davies, J.W. Wilkins, Phys. Rev. Lett., 67, 3720 (1991).

${ }^{12}$ A. Kawabata, J. Phys. Soc. Japan, 60, 3222 (1991).

${ }^{13}$ Y. Meir, N.S. Wingreen, P.A. Lee, Phys. Rev. Lett., 70, 2601 (1993).

14 T.K. Ng, Phys. Rev. Lett., 70, 3635 (1993).

${ }^{15}$ A.L. Yeyati, A. Martin-Rodero, F. Flores, Phys. Rev. Lett., 71, 2991 (1993).

${ }^{16}$ N.S. Wingreen, Y. Meir, Phys. Rev. B, 49, 11040 (1994).

${ }^{17}$ M.H. Hettler and H. Schöller, Phys. Rev. Lett., 74, 4907 (1995). 
18 N. Sivan, N.S. Wingreen, Phys. Rev. B, 54, 11622 (1996).

19 J. König, J. Schmid, H. Schoeller and G. Schön, Phys. Rev. B, 54, 16820 (1996).

20 T.K. Ng, Phys. Rev. Lett., 76, 487 (1996).

${ }^{21}$ A. Schiller and S. Hershfield, Phys. Rev. Lett., 77, 1821 (1996).

${ }^{22}$ H. Shao, D.C. Langreth and P. Nordlander, Phys. Rev. B, 49, 13929 (1994); Phys. Rev. B, 52, 2988 (1995); Phys. Rev. Lett., 77, 948 (1996); P. Nordlander, N.S. Wingreen, Y. Meir, D.C. Langreth, cond-mat/9801241.

${ }^{23}$ Y. Goldin and Y. Avishai, Phys. Rev. Lett., 81, 5394 (1998).

${ }^{24}$ R. Lopez, R. Aguado, G. Platero and C. Tejedor, Phys. Rev. Lett., 81, 4688 (1998).

25 T.C.L.G.Sollner, W.D.Goodhue, P.E.Tannenwald, C.D.Parker, D.D.Peck, Appl. Phys. Lett., 43, 588 (1983).

26 T.C.L.G.Sollner, P.E.Tannenwald, D.D.Peck, W.D.Goodhue, Appl. Phys. Lett., 45, 1319 (1984).

${ }^{27}$ A.Rydberg, H.Grönqvist, Electr. Lett., 25, 348 (1989).

${ }^{28}$ E.R. Brown et al., Appl. Phys. Lett., 54, 934 (1989); Appl. Phys. Lett., 58, 2291 (1991).

${ }^{29}$ One might mistakenly think that contributions from the electric dipole layer can mask the Kondo effect on the time - dependent current. We notice that precisely the same problem exists, in fact, in $D C$ - measurements. The contribution of the Kondo effect to the tunneling current is usually smaller than the background current. It is revealed, then, through a special dependence on the parameters (such as $\ln T$ contribution to the conductance or zero - bias anomaly). Some pertinent effects in the time - dependent current are calculated in the present paper. It therefore provides a direct suggestion for an appropriate experiment. 
${ }^{30}$ L.P. Kouwenhoven, S. Jauhar, K. McCormick, D. Dixon, P.L. McEuen, Yu. V. Nazarov, N.C. van der Vaart, and C.T.Foxon, Phys. Rev. B 50, 2019 (1994).

${ }^{31}$ L.P. Kouwenhoven, S. Jauhar, J. Orenstein, P.L. McEuen, Y. Nagamune, J. Motohisa, and H. Sakaki, Phys. Rev. Lett. 73, 3433 (1994).

${ }^{32}$ R. H. Blick, R. J. Haug, K. von Klitzing, K. Eberl, D.W. van der Weide, Appl. Phys. Lett. 67, 3924 (1995).

33 T. Fujisawa and S. Tarucha, Superlattices Microstructures 21, 247 (1997).

${ }^{34}$ T.H. Oosterkamp, W.G. van der Wiel, S. De Franceschi, C.J.P.M. Harmans and L.P. Kouwenhoven, cond-mat/9904359.

35 J. Kondo, Prog. Theor. Phys., 32, 37 (1964).

36 This is not always true, i.e. for very low temperatures, low bias or strongly asymmetric structures [Y. Goldin, E. Kogan, Y. Avishai and P. Coleman, in preparation], but usually it is the case.

${ }^{37}$ R.K.Tien, J.P.Gordon, Phys. Rev., 129, 647 (1963).

38 J.R.Tucker, IEEE J. Quant. Elec., QE-15, 1234 (1979).

39 J.A. Appelbaum, Phys. Rev. Lett., 17, 91 (1966).

${ }^{40}$ J.A. Appelbaum, Phys. Rev., 154, 633 (1967).

${ }^{41}$ P. Coleman, Phys. Rev. B, 29, 3035 (1984).

${ }^{42}$ A. A. Abrikosov, Physics 2, 5 (1965).

${ }^{43}$ E.L. Wolf, Principles of Electron Tunneling Specroscopy (Oxford University Press, Oxford, 1986), Chap. 8.

44 J. R. Schrieffer, P. A. Wolff, Phys. Rev. 149, 491 (1966). 
${ }^{45}$ C. Bruder, H. Schoeller, Phys. Rev. Lett., 72, 1076 (1994).

${ }^{46}$ G.-L.Ingold, Yu.V.Nazarov, in Single Charge Tunneling, ed. by H.Grabert, M.H.Devoret, NATO ASI Series, Vol. 294 (Plenum Press, New York, 1992).

${ }^{47}$ C. Caroli, R. Combescot, P. Nozieres, D. Saint-James, J. Phys.C: Solid State Phys., 4, $916(1971)$.

${ }^{48}$ S.E. Barnes, J. Phys. F: Metal Phys. 6, 1375 (1976).

${ }^{49}$ N.E. Bickers, Rev. Mod. Phys., 59, 845 (1987).

${ }^{50}$ L.V.Keldysh, Zh. Eksp. Teor. Fiz. 47, 1515 (1964) [Sov. Phys. JETP, 20, 1018 (1965)].

${ }^{51}$ Y.Meir, N.S.Wingreen, Phys. Rev. Lett., 68, 2512 (1992).

${ }^{52}$ N.S.Wingreen, A.P.Jauho, Y.Meir, Phys. Rev. B, 48, 8487 (1993).

53 A.-P. Jauho, N.S. Wingreen and Y. Meir, Phys. Rev. B 50, 5528 (1994).

${ }^{54}$ A.L. Fetter, J.D. Walecka, Quantum Theory of Many - Particle Systems (McGraw - Hill, New York, 1971).

55 J.Schwinger, J. Math. Phys., 2, 407 (1961).

${ }^{56}$ L.P.Kadanoff, G.Baym, Quantum Statistical Mechanics (W.A.Benjamin Inc., New York, 1962).

${ }^{57}$ Z.-B. Su, Lu Yu, L.-Y. Chen, in Thermal Field Theories, edited by H.Ezawa, T.Arimitsu, Y.Hashimoto (North - Holland, Amsterdam, 1991).

${ }^{58}$ K.-C. Chou, Z.-B. Su, B.-L. Hao, Lu Yu, Phys. Rep. (Rev. Section. Phys. Lett), 118, 1 (1985).

${ }^{59}$ L.D. Landau, E.M. Lifshitz, Course of Theoretical Physics (Pergamon Press, New York, 1981), v.10 (Physical Kinetics), p.395. 
${ }^{60}$ G.D. Mahan, Many-Particle Physics, 2nd edition (Plenum Press, New York, 1990).

${ }^{61}$ J. Rammer, H. Smith, Rev.Mod.Phys., 58, 323 (1986).

${ }^{62}$ At moderate external fields (this condition is defined by inequalities (40)), behavior of the Kondo system depends only on the potential difference between the leads, as discussed in the previous section. On the other hand, an application of a uniform (though time dependent) potential is not observable even if it is arbitrary strong and arbitrary fast 63. Therefore, the time - dependent shift of the right lead can be subtracted and only the left lead is considered alternating.

${ }^{63}$ Y.Goldin and Y.Avishai, Phys. Rev. B, 55, 16359 (1997).

${ }^{64}$ It is true also below the Kondo temperature. We explained it in the text for the nonlinear case. In the linear response the function $F(\phi, T, D)$ is linear in $\phi$ by definition. Then it can be exactly proved that only the $D C$ and the first harmonic are generated.

${ }^{65}$ A. Zawadowski, Phys. Rev. Lett. 45, 211 (1980).

${ }^{66}$ K. Vladar, A. Zawadowski, Phys. Rev. B 28, (a) 1564; (b) 1582; (c) 1596 (1983).

${ }^{67}$ J. von Delft, D.C. Ralph, R.A. Buhrman, A.W.W. Ludwig and V. Ambegaokar, Ann. Phys., 263, No. 1, pp. 1-55 (1998); Ann. Phys., 273, pp. 175-241 (1999).

68 D.L. Cox, A. Zawadowski, Advances in Physics, 47, 599 (1998).

${ }^{69}$ A. Muramatsu, F. Guinea, Phys. Rev. Lett. 57, 2337 (1986).

${ }^{70}$ N.S. Wingreen, B.L. Altshuler, Y. Meir, Phys. Rev. Lett. 75, 770 (1995).

${ }^{71}$ K. Yamada et al., Prog. Theor. Phys. 71, 450 (1984).

${ }^{72}$ T. Inoshita et al., Phys. Rev. B 48, 14725 (1993). 


\section{FIGURES}

FIG. 1. a) Schematic drawing of the Kondo peaks in the non-equilibrium interacting density of states of the dot (impurity). Here $\epsilon_{d}$ is the bare energy level of the dot, while $\mu_{L(R)}$ are chemical potentials in the left (right) lead. b) Possible formation of numerous Kondo - resonance peaks in the density of states caused by a time - dependent field. The peaks associated with tunneling to the left and the right leads are schematically shown near the left and the right barriers respectively. $\Omega$ is the frequency of the external field.

FIG. 2. Choice of points on the closed time - path for the external operators in equation (13).

FIG. 3. Diagrams for the perturbation expansion of the current in the Anderson model. Dashed lines stand for slave fermions, dash - dotted lines — for slave bosons, solid lines — for lead electrons. A quantity $s_{i}$ is the number of photons emitted when an electron goes from the dot to a lead, while $q_{i}$ is the number of photons absorbed by an electron going from a lead to the dot.

FIG. 4. Tunneling vertices in the slave - bosons representation of the Anderson model. (a) Tunneling of an electron from the dot to a lead is represented as the decay of a slave fermion into a slave boson and a lead electron. (b) Reverse process representing tunneling from a lead to the dot.

FIG. 5. Choice of points on the closed time - path for the external operators in equation (49).

FIG. 6. Diagrams for the perturbation expansion of the current in the Kondo model. Solid

lines stand for lead electrons, dashed lines - for dot electrons.

FIG. 7. a) Spectrum of the tunneling current for a quantum dot in the Kondo regime (amplitude of the harmonics $I_{n}$ via their number $n$, the value of $1 / 2 I_{0}$ is shown for the dc). The contribution of the second order in $J \rho$ is not shown. The current is measured in units of $C_{3} \cdot k T$. W/ $\Omega=4$, $\Omega / k T=5, \phi^{d c} / k T=10, D / k T=200$. b) Spectrum of the tunneling current for a non - interacting one - level system with the energy level between the chemical potentials of the left and the right leads. The current is measured in units of $e \Gamma / \hbar$, where $\Gamma$ is the level width. $W / \Omega=4, \Omega / \Gamma=5$, $\phi^{d c} / \Gamma=10, D / \Gamma=200$.

FIG. 8. Differential $D C$ - conductance (in units of $C_{3}$ ) versus $D C$ - bias at various values of $A C$

- bias $W$ (both are measured in units of $k T$ ). $\Omega / k T=5, D / k T=200$.

FIG. 9. The same as in the previous plot but at ten - fold lower temperature.

FIG. 10. Differential $A C$ - conductance (in units of $C_{3}$ ) versus $A C$ - bias at various values of $D C$ - bias $\phi^{d c}$ (both are measured in units of $k T$ ). $\Omega / k T=5, D / k T=200$. 
FIG. 11. Derivative of the $A C$ with respect to $D C$ - bias versus the latter (which is given in units of $\hbar \Omega)$. W/ $W=2, D / \Omega=40$. Dashed line is plotted at $\Omega / T=5$, while solid line is at the ten - fold lower temperature. 
a)

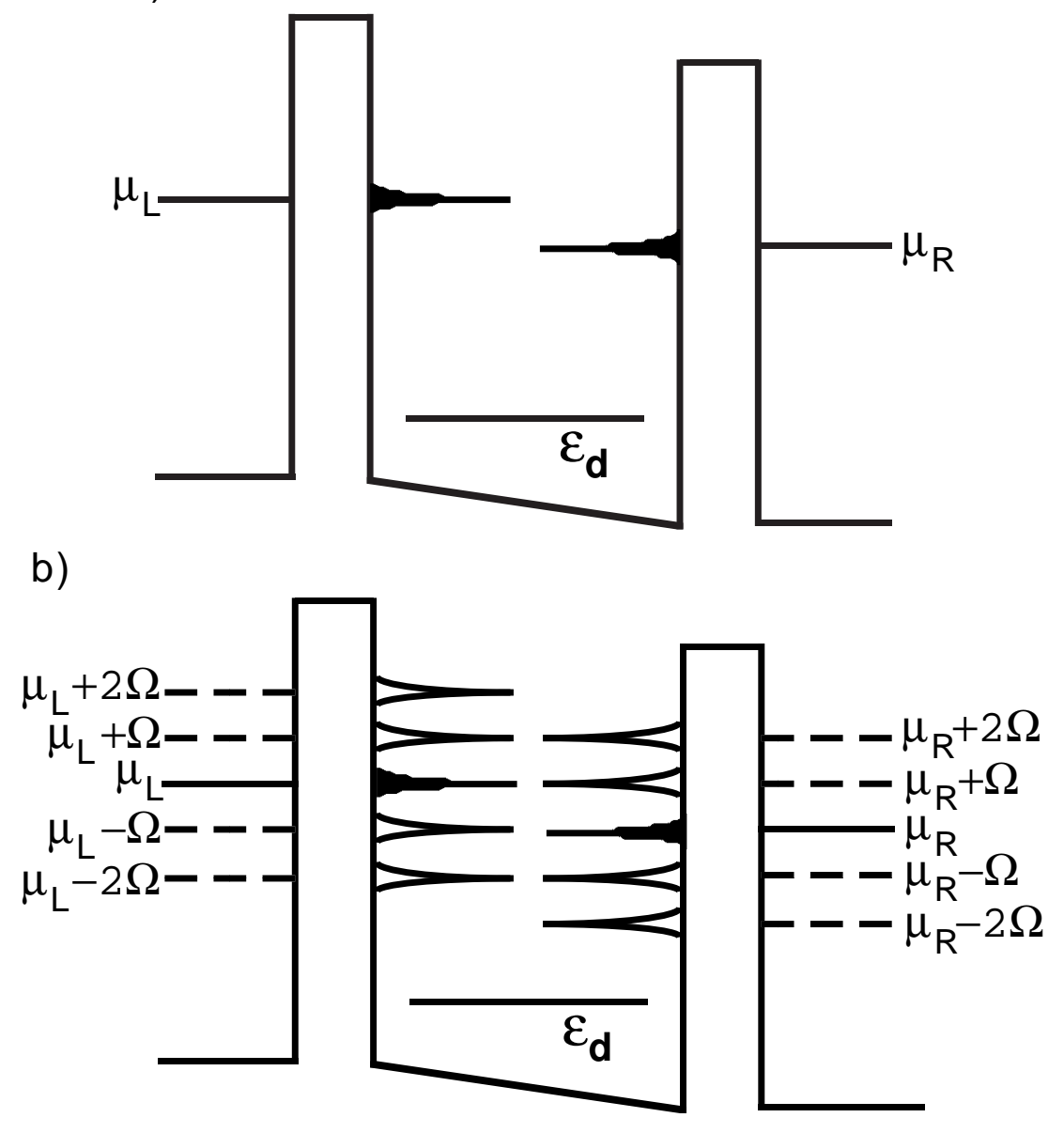

Fig. 1 


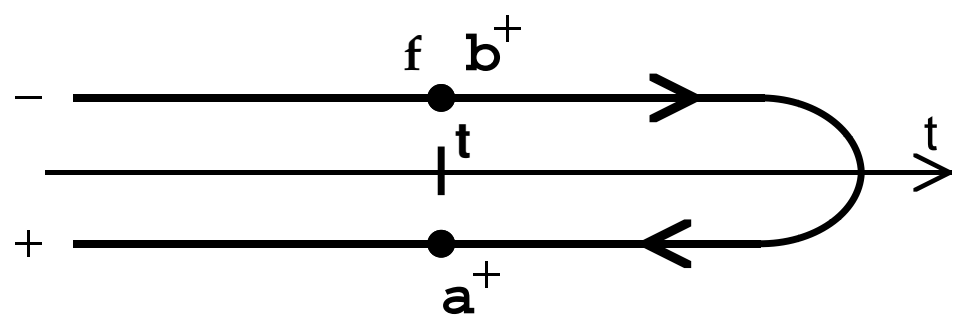

Fig. 2 
a)

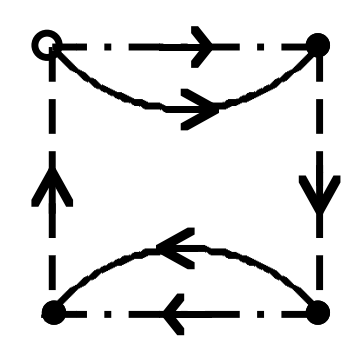

c)

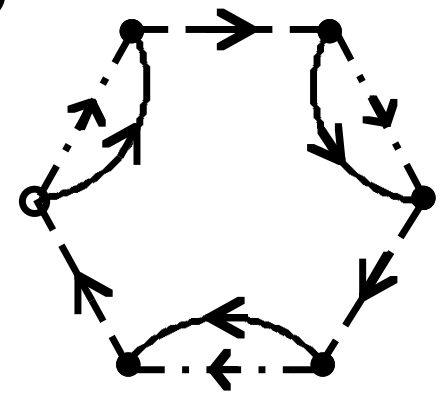

e)

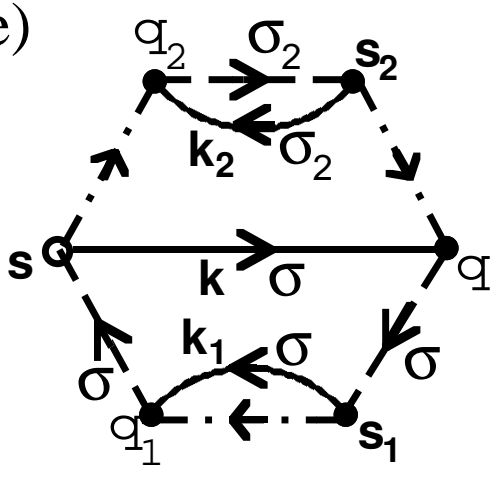

g)

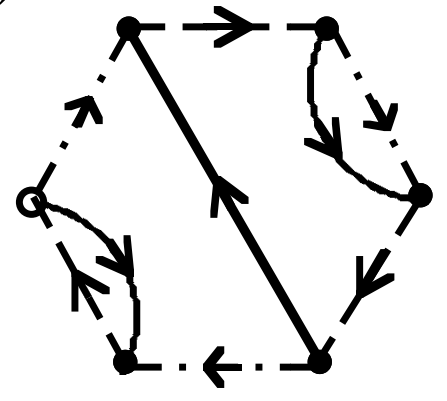

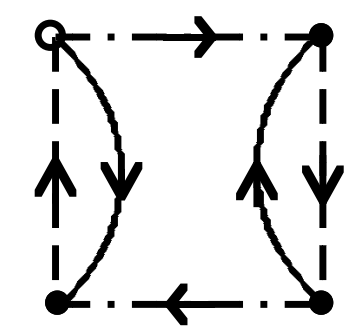

d)

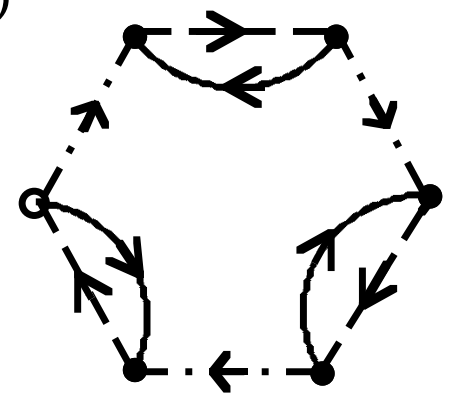

f)

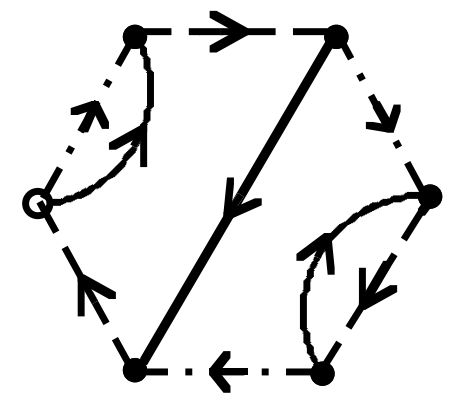

h)

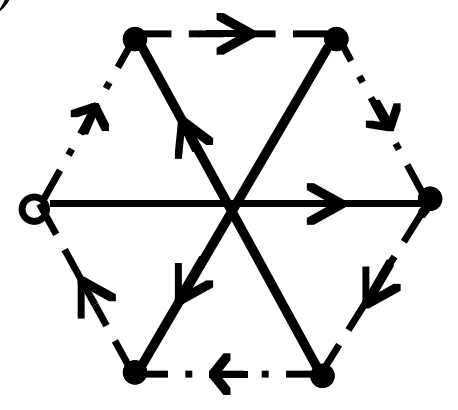

Fig. 3 
a)

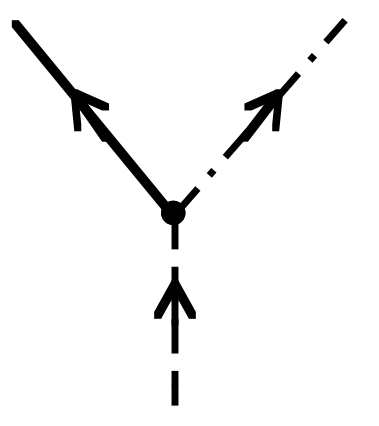

b)

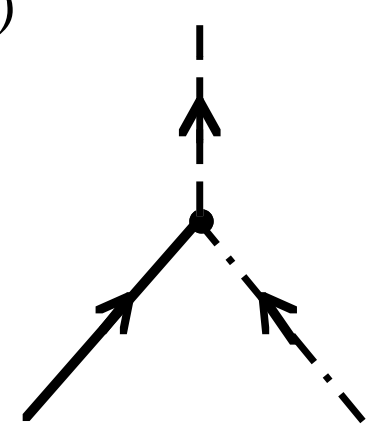

Fig. 4 



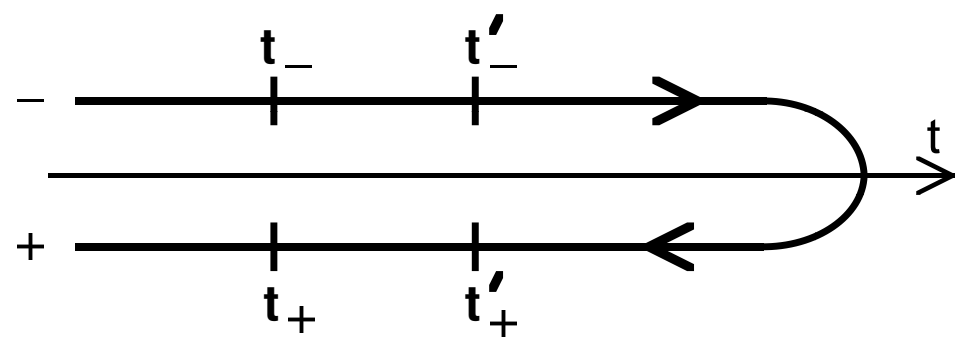

Fig. 5 


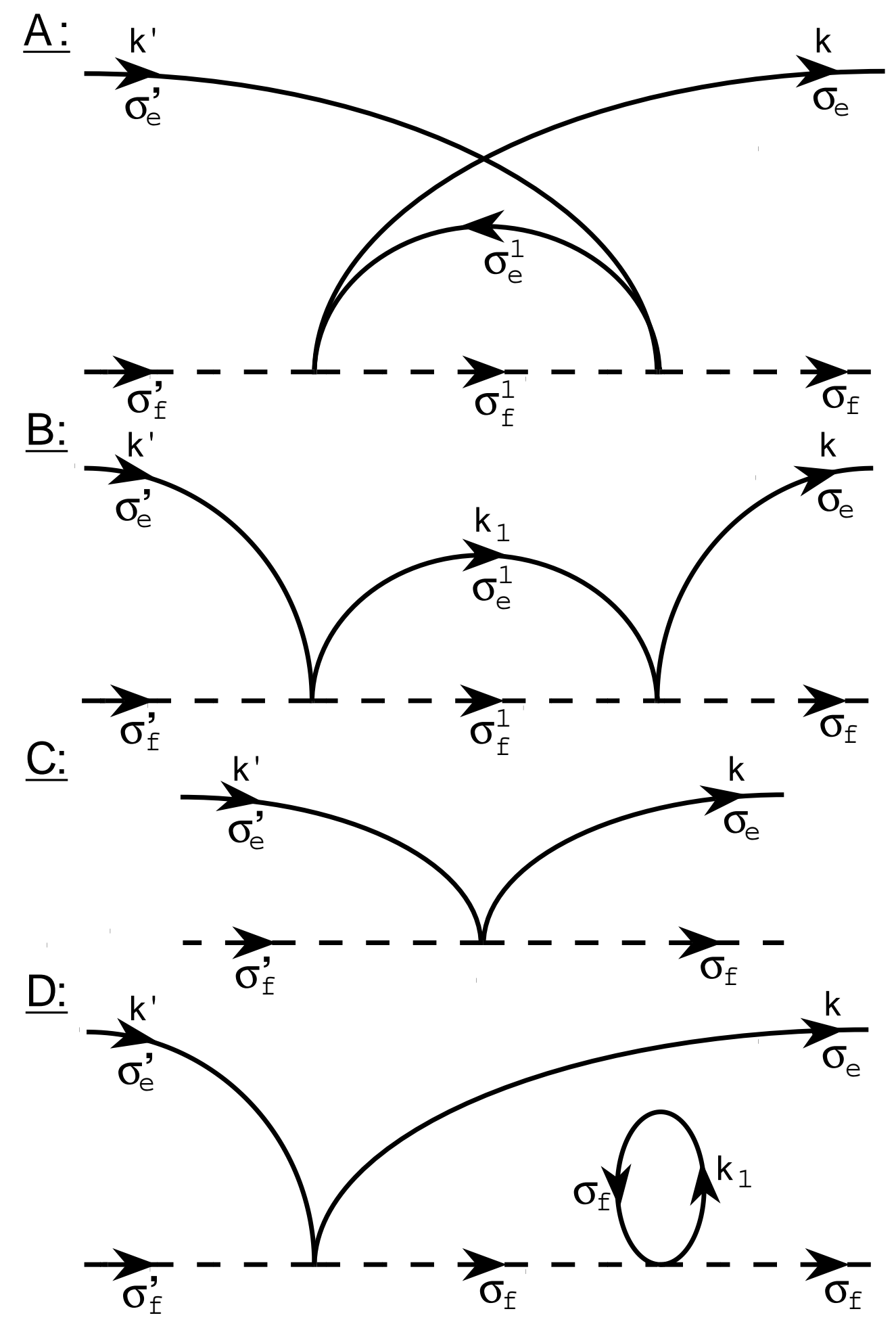

Fig. 6 

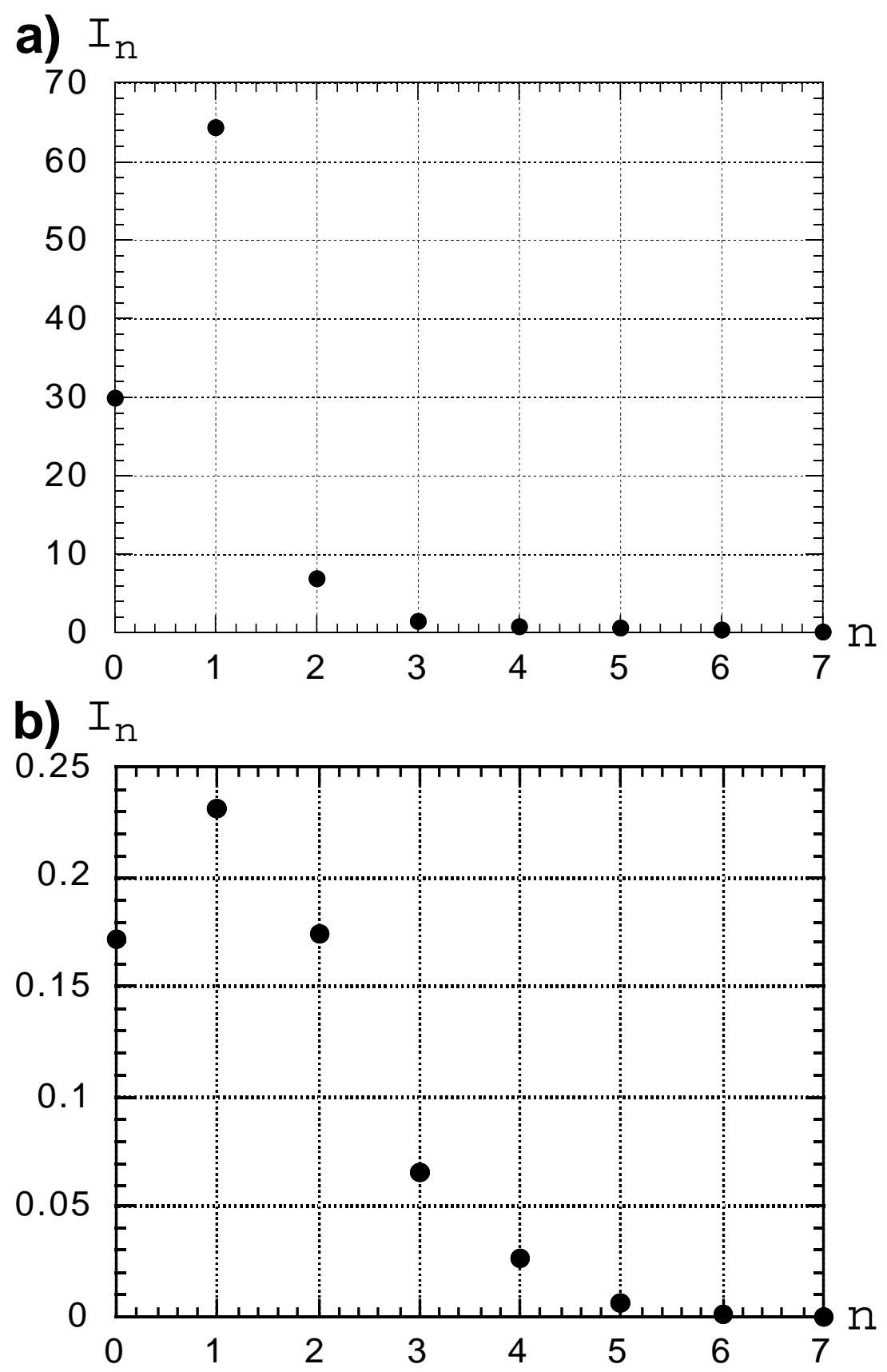

Fig. 7 


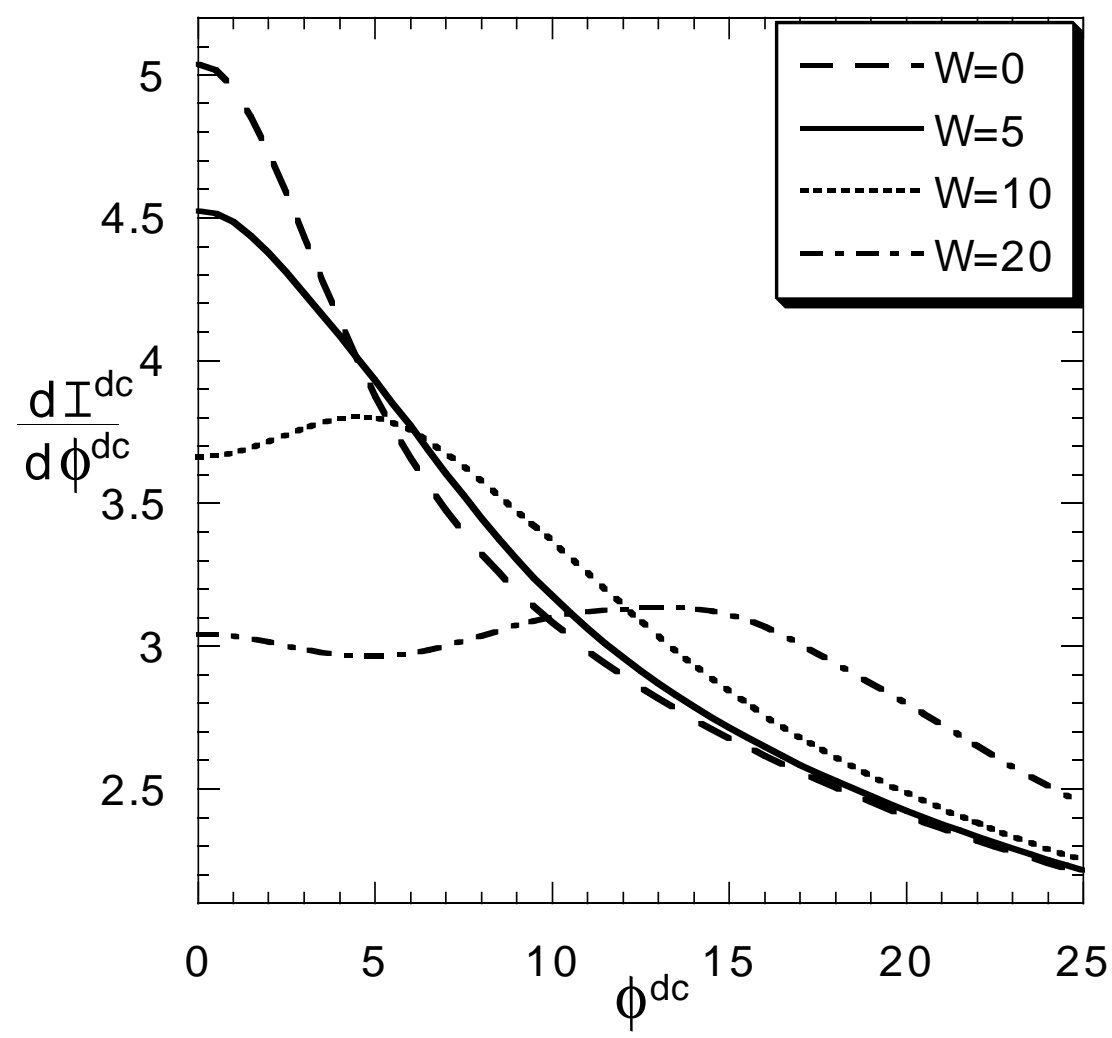

Fig. 8 


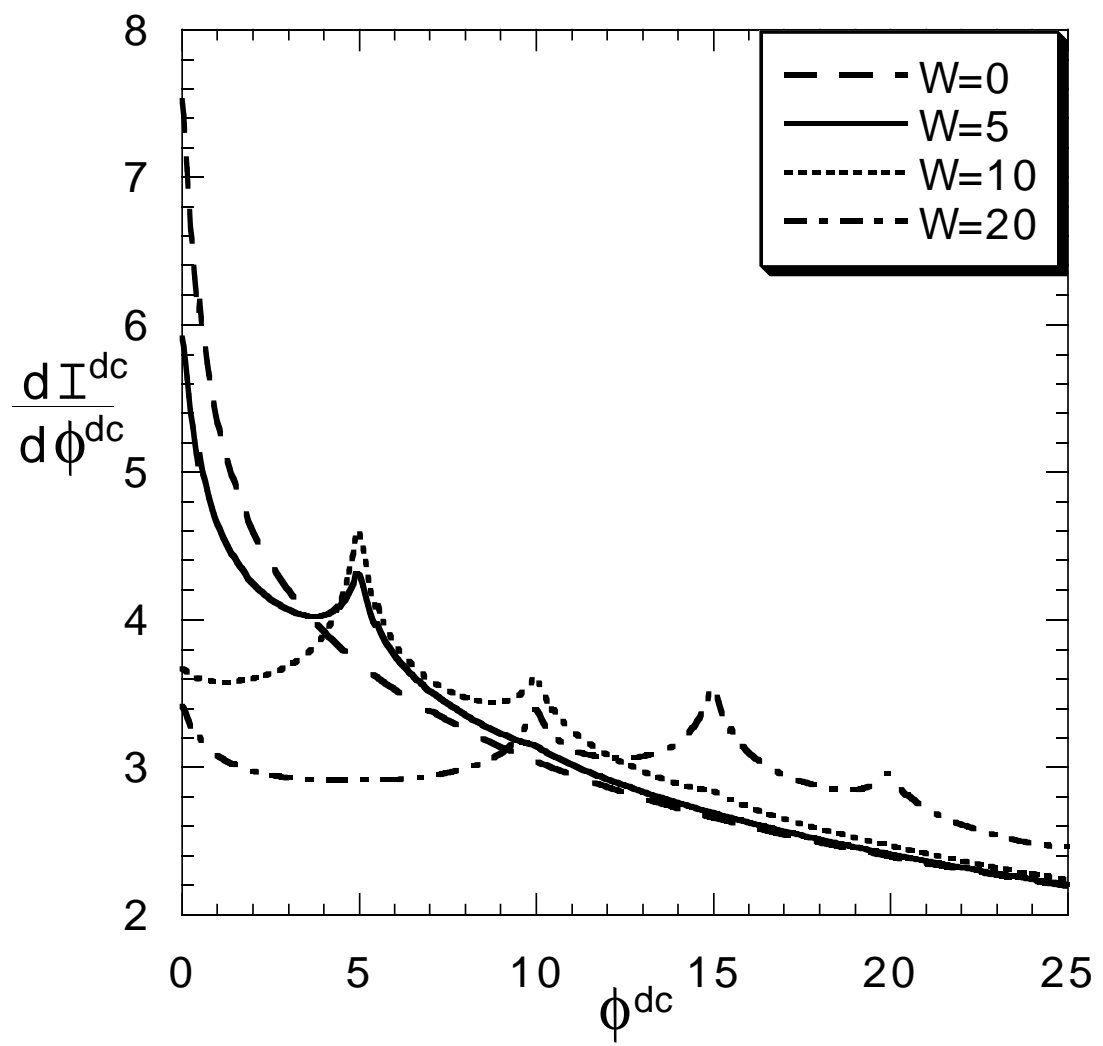

Fig. 9 


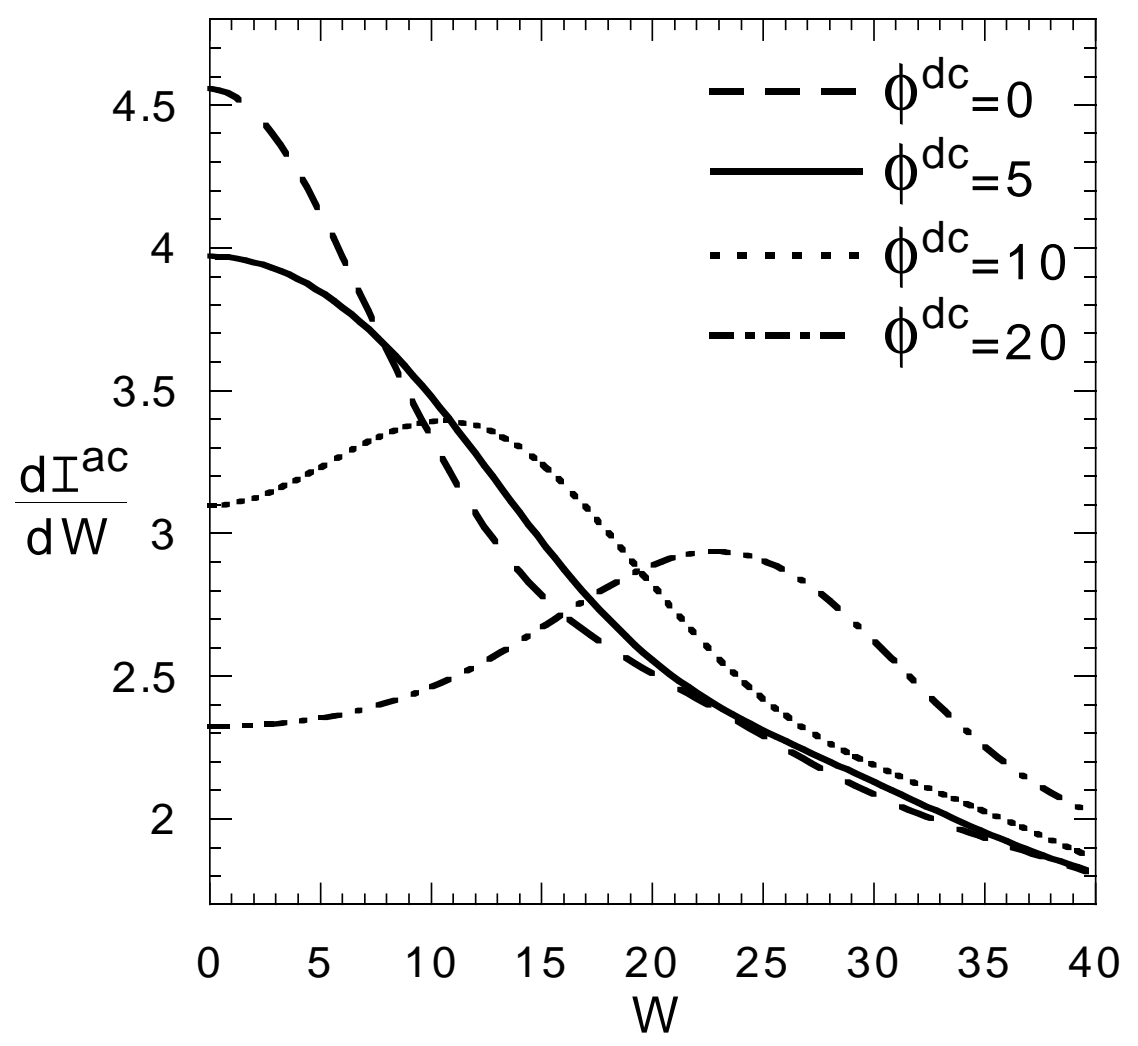

Fig. 10 


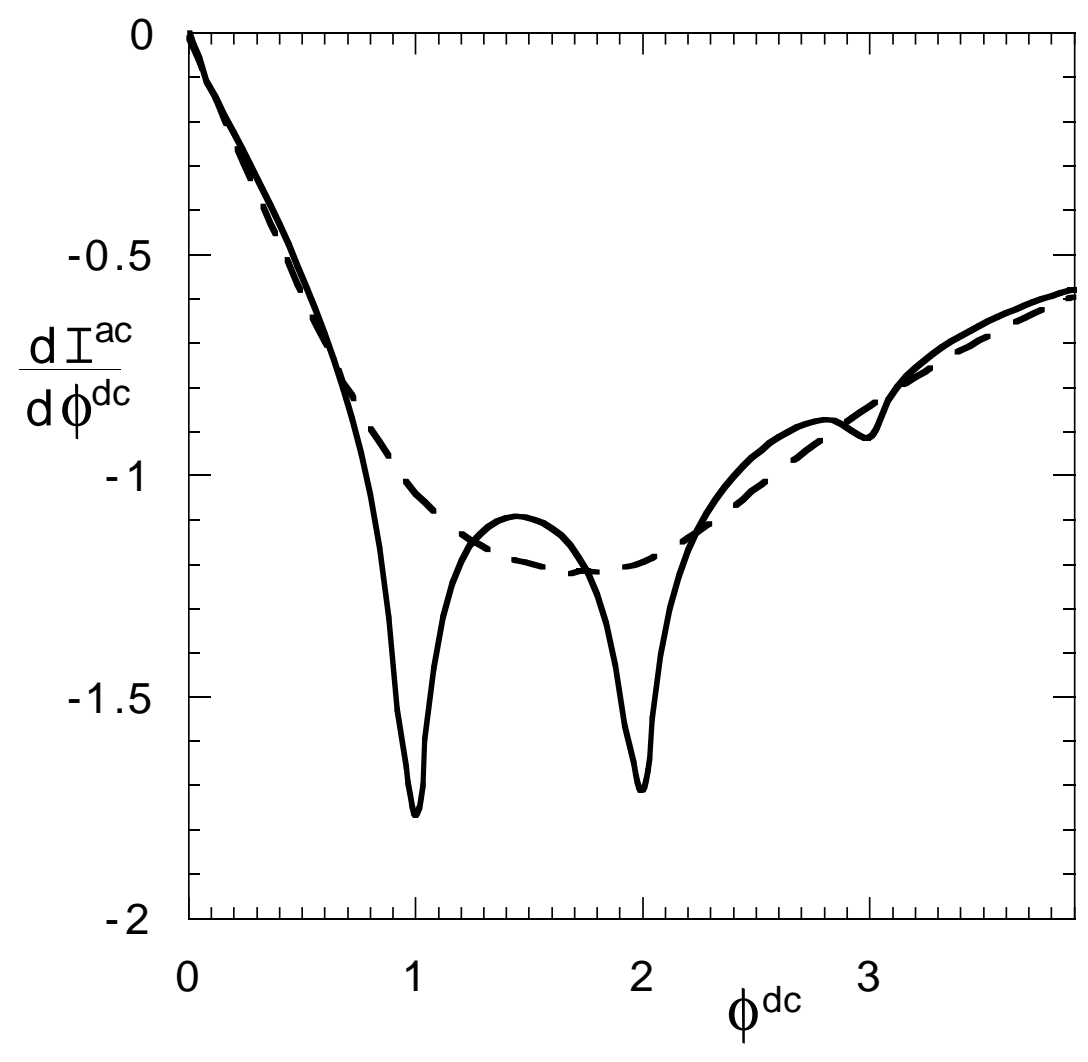

Fig. 11 